TRANSACTIONS OF THE

AMERICAN MATHEMATICAL SOCIETY

Volume 365, Number 6, June 2013, Pages 3041-3068

S 0002-9947(2012)05710-2

Article electronically published on November 28, 2012

\title{
AUTOMORPHISMS OF ALBERT ALGEBRAS AND A CONJECTURE OF TITS AND WEISS
}

\author{
MANEESH THAKUR
}

\begin{abstract}
Let $k$ be a field of characteristic different from 2 and 3. The main aim of this paper is to prove the Tits-Weiss conjecture for Albert division algebras over $k$ which are pure first Tits constructions. The conjecture asserts that, for an Albert division algebra $A$ over a field $k$, the structure group $\operatorname{Str}(A)$ is generated by $U$-operators and scalar multiplications. The conjecture derives its importance from its connections with algebraic groups and Tits buildings, particularly with Moufang polygons. It is known that $k$-forms of $E_{8}$ with index $E_{8,2}^{78}$ and anisotropic kernel a strict inner $k$-form of $E_{6}$ correspond bijectively (via Moufang hexagons) to Albert division algebras over $k$. The Kneser-Tits problem for a form of $E_{8}$ as above is equivalent to the Tits-Weiss conjecture (see Section 3). We provide a solution to the Kneser-Tits problem for $k$-forms of $E_{8}$ corresponding to pure first Tits construction Albert division algebras. As an application, we prove that for the $k$-group $G=\operatorname{Aut}(A), G(k) / R=1$, where $A$ is an Albert division algebra over $k$ as above and $R$ stands for $R$ equivalence in the sense of Manin.
\end{abstract}

\section{INTRODUCTION}

The main result of this paper is a solution to the Tits-Weiss conjecture for Albert division algebras over $k$, which are pure first Tits constructions. As a consequence, we solve the Kneser-Tits problem for $k$-forms of $E_{8}$ with Tits index $E_{8,2}^{78}$, which have anisotropic kernel a strict inner $k$-form of $E_{6}$ associated to pure first construction Albert division algebras. One should note that pure first construction Albert division algebras exist in abundance (see [13]). An Albert division algebra $A$ is a first Tits construction if and only if the anisotropic $k$-form of $E_{6}$ that it corresponds to is split by a degree 3 extension. The Albert algebra is a pure first construction if and only if every degree 3 extension that splits this group is cyclic (see Theorem 2.1). One is guaranteed this situation if, for example, the base field admits only extensions of degree a power of 3 . If $k_{0}$ is an algebraically closed field of characteristic different from 2 and 3, then every Albert division algebra over the iterated Laurent series field $L=k_{0}\left(\left(x_{1}, \ldots, x_{n}\right)\right), n \geq 3$ is a pure first construction (see [13]).

Let $G$ be a semisimple, simply connected algebraic group, defined and isotropic over a field $k$. Let $G(k)$ denote the group of $k$-rational points of $G$ and $G(k)^{+}$be the normal subgroup of $G(k)$, generated by the $k$-rational points of the unipotent

Received by the editors July 16, 2011 and, in revised form, September 22, 2011.

2010 Mathematics Subject Classification. Primary 20G15; Secondary 17C30.

Key words and phrases. Automorphisms, Albert algebras, structure group, inner structure group, Kneser-Tits. 
radicals of the parabolic $k$-subgroups of $G$. The Kneser-Tits problem asks if the quotient $W(k, G)=G(k) / G(k)^{+}$is trivial (see [19], 7.2 and [4 for a recent survey). In this paper we answer this question in the affirmative for a certain rank 2 form of $E_{8}$. The form of $E_{8}$ that interests us has Tits index $E_{8,2}^{78}$ and has anisotropic kernel a strict inner form of $E_{6}$. Such groups exist over $k$ if $k$ admits central division algebras of degree 3 with nonsurjective reduced norm map or such algebras with unitary involutions over a quadratic extension of $k$ (see [27]).

The Kneser-Tits problem for the form of $E_{8}$ mentioned above has a reformulation in terms of the structure group of an Albert division algebra. This reformulation is due to J. Tits and R. Weiss, which made its first appearance in their book on Moufang polygons (see [31, 37.41, 37.42 and page 418), in the form of a conjecture, henceforth referred to as the Tits-Weiss conjecture (this is explained ahead). The assertion of the conjecture makes sense for reduced Albert algebras as well and follows rather easily from known results of Jacobson (see Theorem 5.12). Albert algebras are of profound importance in Lie theory and the theory of buildings. Exceptional groups of types $F_{4}, E_{6}, E_{7}$ and $E_{8}$ have close links with Albert algebras (see [3], 6], 23], [31]). For example, over a base field $k$, all groups of type $F_{4}$ arise as groups of automorphisms of Albert algebras. Let $A$ be an Albert algebra over $k$ containing no nonzero nilpotents and let $G=\operatorname{Aut}(A)$ denote the algebraic group of automorphisms of $A$. Then $G$ is defined and anisotropic over $k$, of type $F_{4}$ (for a proof, see [20], Lemma 3.1).

We study some group-theoretic properties of the structure group and the group of automorphisms of an Albert algebra over a given field, which we describe briefly. Every Albert algebra $A$ over a field $k$ comes equipped with a cubic form $N$, called the norm form of $A$, and a linear form $T$ defined on it, called its trace form. Every automorphism of $A$ leaves these forms invariant. A norm similarity of $A$ is a bijective linear map $\theta: A \rightarrow A$ such that $N(\theta(x))=\alpha N(x)$ for all $x \in A$, where $\alpha \in k^{*}$ is independent of $x$, and this identity should hold over all base field extensions. We call $\alpha$ the factor of similitude of $\theta$. A norm similarity with factor of similitude 1 is called a norm isometry.

It is well known that the group of norm isometries of $A$ is a simply connected group of type $E_{6}$ defined over $k$ (see [23, Theorem 7.3.2). The isotropy subgroup of $1 \in A$ in the group of isometries is precisely the group of automorphisms of $A$ (a $k$ group of type $F_{4}$ ). Clearly any scalar multiplication $R_{t}, t \in k^{*}$ is a norm similarity, since $N\left(R_{t}(x)\right)=N(t x)=t^{3} x$ for all $x \in A$. The right multiplications $R_{a}, a \in A^{*}$, by elements of $A$, however, are not similarities of the norm in general. The linear operators $U_{a}$ for $a \in A$ defined by $U_{a}=2 R_{a}^{2}-R_{a^{2}}$ (these have a valid expression in characteristic 2 as well; see Section 5 ) are norm similarities with similitude factor $N(a)^{2}$ if $a$ is invertible in $A$ (see [6], Chapt. VI, Section 7).

For an invertible element $u \in A$, the $u$-isotope of $A$ is the algebra $A^{(u)}$ whose underlying vector space structure is the same as $A$ but the multiplication on $A^{(u)}$ is defined as $x_{u} y=\{x u y\}$, where $\{x y z\}$ is the Jordan triple product in $A$, given by

$$
\{x y z\}=U_{x, z}(y), U_{x, z}=R_{x} R_{z}+R_{z} R_{x}-R_{x z}
$$

where $R_{x}$ denotes the right multiplication on $A$ by $x$. Note that $U_{a}=U_{a, a}$. Two Albert algebras $A$ and $A^{\prime}$ are said to be isotopic if there is an isomorphism $\phi$ : $A^{(u)} \longrightarrow A^{\prime}$ for some invertible element $u \in A$. Such an isomorphism is called an 
isotopy from $A$ to $A^{\prime}$. The set of all isotopies from $A \longrightarrow A$ forms a group under composition of maps, called the structure group of $A$, denoted by $\operatorname{Str}(A)$. It is known that isotopies are the same as the norm similarities as long as the base field is not too small (see 6], Chap.VI, Theorem 6, Theorem 7). The subgroup of $\operatorname{Str}(A)$ generated by the $U_{a}, a$ invertible, is called the inner structure group of $A$, denoted by $\operatorname{Instr}(A)$. It follows that $\operatorname{Instr}(A)$ is a normal subgroup of $\operatorname{Str}(A)$ (see [31], 37.42). Let $C$ be the subgroup of $\operatorname{Str}(A)$ consisting of the $R_{t}, t \in k^{*}$. Then $C$.Instr $(A)$ is normal in $\operatorname{Str}(A)$. We can now state the conjecture of Tits and Weiss (see [31], page 418):

Conjecture (Tits-Weiss). Let $A$ be an Albert division algebra over a field $k$. With the notation introduced above,

$$
\frac{\operatorname{Str}(A)}{\operatorname{C.Instr}(A)}=\{1\}
$$

This conjecture has origins in the theory of Moufang polygons and is of fundamental importance to the subject. In this paper, we settle this conjecture for Albert division algebras that are pure first Tits constructions, thereby solving the Kneser-Tits problem for the $k$-forms of $E_{8}$ arising from such Albert division algebras.

The elements of $\operatorname{Aut}(A) \cap \operatorname{Instr}(A)$ are called inner automorphisms of $A$. We prove that every automorphism of an Albert division algebra which is a pure first Tits construction is inner (Corollary [5.5). The strategy to prove the above conjecture is to reduce the assertion about norm similarities to an assertion about automorphisms. The main step is to prove that every automorphism is inner. The key tool is a fixed point theorem for automorphisms of Albert algebras. We prove that every automorphism of an arbitrary Albert algebra $A$ fixes a nonzero element with trace zero. When the Albert algebra is a division algebra, this proves that every automorphism fixes a cubic subfield pointwise (Corollary 4.2). We reprove a theorem of Jacobson on automorphisms of Cayley algebras and then use the same idea to derive the result on automorphisms of Albert division algebras.

We apply our results on automorphisms of Albert division algebras to prove the triviality of yet another group, related to $R$-equivalence. Let $A$ be an Albert division algebra over $k$ that is a pure first construction and $G=\operatorname{Aut}(A)$. We prove that $G(k) / R=1$, where $R$-stands for $R$-equivalence in algebraic groups (see [1]).

Groups with index $E_{8,2}^{78}$ : Let $G$ be a semisimple simply connected group defined over $k$ with Tits index $E_{8,2}^{78}$ (see [29] for the index notation) and anisotropic kernel a strict inner form of $E_{6}$. Let $\Gamma$ denote the building associated to $G$, in the sense of 28] or [31. It is shown in [31] that $\Gamma$ is a Moufang hexagon, defined by a hexagonal system of type $27 / F$, where $F / k$ is either a quadratic extension of $k$ or $F=k$. Recall from [31, 42.3.5, 42.6] that such forms of $E_{8}$ exist if and only if $k$ admits Albert division algebras. Albert division algebras over $k$ exist if and only if there are degree 3 central division algebras over $k$ with nonsurjective reduced norm maps, or $k$ admits degree 3 central division algebras with unitary involution, over a quadratic extension of $k$, with nonsurjective reduced norm map. Hence these forms of $E_{8}$, for example, do not exist over finite fields, number fields, local fields or the field of real numbers; however, the rational function field $\mathbb{Q}(t)$ admits these groups. 
In this context the following theorem of Albert is of importance (see [6], Theorem 21, Chap. IX):

Theorem 1.1. Let $k$ be a field such that there are degree 3 central division algebras over $k$. Then there exist Albert division algebras over $k(t)$, the function field in one indeterminate.

Contrast this with the fact that $k=\mathbb{C}\left(\left(x_{1}, \ldots, x_{n}\right)\right), n \geq 3$, admits Albert division algebras (see [13]), even though there are no central division algebras of degree 3 over $\mathbb{C}$.

Moufang hexagons of type 27/F : Moufang hexagons of type $27 / F$ correspond to Albert division algebras over $k$. Let $\Gamma$ be the Moufang hexagon associated to an Albert division algebra $A$ over $k$, as described in Chapter 15 of [31], or 4.7 of [28]; see also [25] for an excellent introduction to the subject. Let $G$ be the group of "linear" automorphisms of $\Gamma$ (see Section 3) and let $G^{\dagger}$ be the subgroup generated by the root groups of $\Gamma$. Then $G^{\dagger}$ is a normal subgroup of $G$ and the quotient $G / G^{\dagger}$ is isomorphic to $H / H^{\dagger}$, where $H$ is the structure group of the Albert algebra $A$ and $H^{\dagger}$ is the subgroup of $G$ generated by all maps of the form $x \mapsto t x, t \in k^{*}$ and $x \mapsto U_{b}(x), b \in A^{*}$; this is proved in 37.41 of [31. The group $G$ is the group of $k$ rational points of an algebraic group defined over $k$ with index $E_{8,2}^{78}$, with anisotropic kernel $H$, the structure group of $A$. By 42.3.6 of [31, the root groups of $\Gamma$ are the groups $\mathcal{U}_{\alpha}(k)$, where $\alpha$ is a nondivisible root relative to a maximal $k$-split torus $S$ and $\mathcal{U}_{\alpha}$ is the corresponding unipotent $k$-group. This explains the Kneser-Tits problem in the context of the Tits-Weiss conjecture stated in [31, page 418] for the structure group of $A$.

$R$-equivalence, rationality and the Whitehead group: An irreducible algebraic variety $X$ over a field $k$ is said to be $k$-rational if $X$ is birationally isomorphic over $k$ to an affine space. The rationality problem for algebraic groups is of interest for its connections with some well-known problems, e.g. the Kneser-Tits problem described above. Groups of small ranks are known to be rational. More precisely, let $G$ be a semisimple algebraic group defined over $k$. Then $G$ is $k$-rational if rank $G \leq 2$ (see 2]).

For the notion of $R$-equivalence, we refer to the book [32, Chapter 6] or the book [11, Chapter II, 14]. Let $X$ be an irreducible variety over a field $k$, with $X(k)$ nonempty. We call points $x, y \in X(k) R$-equivalent if there exist points $x=x_{0}, x_{1}, \ldots, x_{n}=y \in X(k)$ and rational maps $f_{i}: \mathbb{A}^{1} \rightarrow X, 1 \leq i \leq n$, all defined over $k$ and regular at 0 and 1 , such that $f_{i}(0)=x_{i-1}, f_{i}(1)=x_{i}$. In case $X=G$, a connected algebraic group, the set of elements of $G(k)$ which are $R$-equivalent to $1 \in G(k)$ is a normal subgroup $R G(k)$ of $G(k)$. The quotient $G(k) / R G(k)$ is denoted by $G(k) / R$. If $G$ is $k$-rational, then one has $G(k) / R=1$ (see [4]).

Now let $G$ be a connected reductive group defined and isotropic over $k$ and $G(k)^{+}$be the subgroup of $G(k)$ generated by the $k$-rational points of the unipotent radicals of the parabolic $k$-subgroups of $G$. This is a normal subgroup of $G(k)$. The quotient $W(k, G)=G(k) / G(k)^{+}$is called the Whitehead group of $G$. If $G$ is simply connected, the Kneser-Tits problem asks if $W(k, G)=1$. 
Let $G$ be a semisimple simply connected and absolutely almost simple group defined and isotropic over $k$. Then one has an isomorphism (see [4])

$$
W(k, G) \simeq G(k) / R
$$

In this paper, we discuss these relations for groups with $k$-index $E_{8,2}^{78}$. We show $R$-triviality for certain groups that are adjoint of type $E_{6}$, occurring as anisotropic kernels of the groups of type $E_{8}$ as mentioned. We also get $R$-triviality for the corresponding $k$-form of $F_{4}$, more or less straight from the triviality of the Whitehead group for the form of $E_{8}$.

Remark on characteristic restrictions: Though in the paper we work over a base field of characteristic different from 2 and 3, by Remark 7.4 in [4, we get results in all characteristics, as far as triviality of Whitehead groups and the Kneser-Tits problem are concerned. It seems likely that the results on norm similarities and automorphisms also hold for arbitrary characteristics.

Structure of the paper: In the paper, some of the results are valid over arbitrary characteristic; however, for uniformity, we will work over a base field $k$ of characteristic different from 2 and 3 . We now describe the paper briefly.

In Section 2, we introduce the notion of an Albert algebra and describe Tits constructions of such algebras, which will be used in the sequel. Section 3 gives preliminary material on Moufang hexagons of type $27 / F$ and relates the KneserTits problem to the Tits-Weiss conjecture. In Section 4, we take up the study of automorphisms of Albert algebras. The main tool in this is the fixed point subalgebra of an automorphism or, more generally, of a given (algebraic) subgroup of the algebraic group of automorphisms $\operatorname{Aut}(A)$ of a given Albert algebra $A$ over $k$. The results of Section 5 are oriented towards a proof of the Tits-Weiss conjecture. This section depends very little on Section 4 , the key result being that every automorphism of an Albert division algebra, which is a pure first construction, is inner (i.e. is in the inner structure group of $A$ as defined in the introduction). This is proved step by step, while proving some results valid for general first constructions.

We use a theorem of Wang on the reduced Whitehead group of division algebras (33], 30], Proposition 2.7) to settle the Tits-Weiss conjecture. We also derive some results for the norm similarities of first constructions, which help us in the proof. Using an analogue of Wang's result for the unitary Whitehead group of a division algebra, due to Yanchevskii (cf. [10, Proposition 17.27), we prove that some automorphisms of a second Tits construction Albert algebra are inner (Corollary 5.4).

We apply the results of Section 5 to the $R$-triviality problem for groups of type $F_{4}$ over $k$, arising from pure first construction Albert division algebras.

\section{Albert algebras}

In this section, we introduce some basic material on Albert algebras over a field $k$, assumed to be of characteristic not 2 or 3 for uniformity of exposition. We assume some familiarity with the notion of an octonion (Cayley) algebra, which can be found in [23] for example. For a detailed account of Albert algebras and octonion algebras we refer to [6], 10], or [23]. 
Reduced Albert algebras: Let $C$ be an octonion algebra over $k$ and let $\Gamma=$ $\operatorname{Diag}\left(\gamma_{1}, \gamma_{2}, \gamma_{3}\right) \in G L(3, k)$ be a diagonal matrix. Let $x \mapsto \bar{x}$ denote the standard involution on $C$. Let $*: M_{3}(C) \rightarrow M_{3}(C)$ denote the involution $X \mapsto X^{*}=$ $\Gamma^{-1} \bar{X}^{t} \Gamma$, where $M_{3}(C)$ denotes the (nonassociative) ring of $3 \times 3$ matrices with entries from $C$ and $\bar{X}=\left(\overline{x_{i j}}\right)$ for $X=\left(x_{i j}\right)$. Let $\mathcal{H}\left(M_{3}(C), *\right)=\mathcal{H}_{3}(C, \Gamma)$ denote the space of hermitian (i.e. *-symmetric) matrices in $M_{3}(C)$. Then clearly $\operatorname{Dim}_{k}\left(\mathcal{H}_{3}(C, \Gamma)\right)=27$. We define a product on this space by $X \cdot Y=\frac{1}{2}(X Y+Y X)$. We thus get a $k$-algebra structure, called a reduced Albert algebra over $k$. These algebras have zero divisors. We call $A=\mathcal{H}_{3}(C, \Gamma)$ split if $C$ is split over $k$. It turns out that there is a unique split Albert algebra over a field $k$, up to isomorphism.

An Albert algebra over $k$ can be defined as a $k$-algebra $A$ such that $A$ is isomorphic to the split Albert algebra over some field extension of $k$. An Albert algebra in which all nonzero elements are invertible, i.e. have their $U$-operators bijective, is called an Albert division algebra. The automorphism group of the split Albert algebra over $k$ is the split form of $F_{4}$ over $k([10,25.13)$. It is known that the automorphism group of an Albert algebra is a $k$-anisotropic form of $F_{4}$ if and only if the algebra has no nonzero nilpotents (cf. for example [20], Lemma 3.1, for a proof). In particular, the automorphism group of an Albert division algebra is a $k$-anisotropic form of $F_{4}$.

For a reduced Albert algebra, there is a natural notion of the trace, denoted $x \mapsto T(x)$; namely, the trace of a matrix in a reduced Albert algebra $A$ is the sum of its diagonal entries (which are scalars) and is a linear form on $A$. Similarly, there is a norm defined on $A$, denoted $x \mapsto N(x)$, which is the "determinant" computed in a suitable way, and is a cubic form on $A$. The notions of trace and norm pass down to an arbitrary Albert algebra over $k$. It is known that any Albert algebra over a field is either reduced or is a division algebra. Any Albert algebra $A$ comes equipped with a quadratic map $\sharp: A \rightarrow A$, written as $x \mapsto x^{\sharp}$, that ties up with the norm map via the identity $N(x)=x x^{\sharp}=x^{\sharp} x$ for all $x \in A$. We call $x^{\sharp}$ the adjoint of $x$. An Albert algebra is a division algebra if and only if its norm is anisotropic over $k$, i.e. has no nontrivial zeros over $k$ (see [6]).

Tits has given two general constructions of all Albert algebras over a field $k$. It is known that any Albert algebra over $k$ comes from either of the two constructions. We shall give formulae for the norm, the trace and the adjoint in the two constructions below. We now describe them briefly.

\section{Tits constructions of Albert algebras}

The first construction: Let $k$ be a base field as fixed before. Let $D$ be a central simple (associative) algebra over $k$ of degree 3 . We will denote by $D_{+}$the (special) Jordan algebra structure on $D$, with multiplication $x \cdot y=\frac{1}{2}(x y+y x), x, y \in D$. Let $\mu \in k^{*}$ be a scalar. Let $N_{D}$ and $T_{D}$ respectively denote the reduced norm and reduced trace maps on $D$. To this data, one associates an Albert algebra $J(D, \mu)$ as follows:

$$
J(D, \mu)=D_{0} \oplus D_{1} \oplus D_{2},
$$

where $D_{i}, i=0,1,2$, is a copy of $D$. For the multiplication on $J(D, \mu)$, we need more notation. For $a, b \in D$ define

$$
a \cdot b=\frac{1}{2}(a b+b a), a \times b=2 a \cdot b-T_{D}(a) b-T_{D}(b) a+\left(T_{D}(a) T_{D}(b)-T_{D}(a \cdot b)\right)
$$


and $\widetilde{a}=\frac{1}{2}\left(T_{D}(a)-a\right)$. The multiplication on $J(D, \mu)$ is given by the formula:

$$
\begin{aligned}
&\left(a_{0}, a_{1}, a_{2}\right)\left(b_{0}, b_{1}, b_{2}\right) \\
&=\left(a_{0} \cdot b_{0}+\widetilde{a_{1} b_{2}}+\widetilde{b_{1} a_{2}}, \widetilde{a_{0}} b_{1}+\widetilde{b_{0}} a_{1}+(2 \mu)^{-1} a_{2} \times b_{2}, \widetilde{a_{2}} \widetilde{b_{0}}\right. \\
&\left.+b_{2} \widetilde{a_{0}}+\frac{1}{2} \mu a_{1} \times b_{1}\right) .
\end{aligned}
$$

It is known that $J(D, \mu)$ is an Albert algebra over $k$ (see [10] or [23] for more details). Further, $J(D, \mu)$ is a division algebra if and only if $\mu$ is not a reduced norm from $D$. Clearly $D_{+}$is a subalgebra of $J(D, \mu)$. Let $A$ be an Albert algebra over $k$ and let $D_{+} \subset A$ for some degree 3 central simple algebra $D$. Then there exists $\mu \in k^{*}$ such that $A \simeq J(D, \mu)$.

Trace and norm maps: Let $A=J(D, \mu)$ be as defined above. The trace $T$ and the norm $N$ on $A$ are given by the formulae:

$$
T(x, y, z)=T_{D}(x), N(x, y, z)=N_{D}(x)+\mu N_{D}(y)+\mu^{-1} N_{D}(z)-T_{D}(x y z) .
$$

From this, one gets an expression for the trace bilinear form on $A$, defined by $T(x, y)=T(x y), x, y \in A$. Therefore, one has, for $x=\left(x_{0}, x_{1}, x_{2}\right), y=\left(y_{0}, y_{1}, y_{2}\right)$,

$$
T(x, y)=T_{D}\left(x_{0} y_{0}\right)+T_{D}\left(x_{1} y_{2}\right)+T_{D}\left(x_{2} y_{1}\right) .
$$

One knows that an Albert algebra $A$ is a division algebra if and only if its norm form is anisotropic over $k$ (see [6]).

The adjoint map: Let $A=J(D, \mu)$. One defines the adjoint map on $A$ as follows. Let $x=\left(x_{0}, x_{1}, x_{2}\right)$. We define

$$
x^{\#}=\left(x_{0}^{\#}-x_{1} x_{2}, \mu^{-1} x_{2}^{\#}-x_{0} x_{1}, \mu x_{1}^{\#}-x_{2} x_{0}\right),
$$

where, for $y \in D, 2 y^{\#}=y \times y$ describes the usual adjoint map on $D$. One can prove that $x x^{\#}=x^{\#} x=N(x)$ for all $x \in A$ (see [6], [10] for details).

The second construction: Let $K / k$ be a quadratic extension and let $(B, \tau)$ be a central simple $K$-algebra of degree 3 over $K$ with a unitary involution $\tau$ over $K / k$. Let $u \in B^{*}$ be such that $\tau(u)=u$ and $N_{B}(u)=\mu \bar{\mu}$ for some $\mu \in K^{*}$; here the bar denotes the nontrivial $k$-automorphism of $K$, and $N_{B}$ is the reduced norm map on $B$. Let $\mathcal{H}(B, \tau)$ be the special Jordan algebra structure on the $k$-vector subspace of $B$ of $\tau$-symmetric elements in $B$, with multiplication as in $B_{+}$. Let $J(B, \tau, u, \mu)=\mathcal{H}(B, \tau) \oplus B$. With the notation introduced above, we define a multiplication on $J(B, \tau, u, \mu)$ by

$$
\left(a_{0}, a\right)\left(b_{0}, b\right)=\left(a_{0} \cdot b_{0}+\widetilde{a u \tau(b)}+\widetilde{b u \tau(a)}, \widetilde{a_{0}} b+\widetilde{b_{0}} a+\bar{\mu}(\tau(a) \times \tau(b)) u^{-1}\right) .
$$

Then $J(B, \tau, u, \mu)$ is an Albert algebra over $k$ and is a division algebra if and only if $\mu$ is not a reduced norm from $B$. Clearly $\mathcal{H}(B, \tau)$ is a subalgebra of $J(B, \tau, u, \mu)$. It is known that if $\mathcal{H}(B, \tau)$ is a subalgebra of an Albert algebra $A$ over $k$, then there are suitable parameters $u \in B^{*}$ and $\mu \in K^{*}$, where $K$ is the centre of $B$, such that $A \simeq J(B, \tau, u, \mu)($ see [6], [10] $)$. 
Trace and norm maps: Let $A=J(B, \tau, u, \mu)$ be an Albert algebra arising from the second construction. The trace $T$ and the norm $N$ on $J(B, \tau, u, \mu)$ are given by the formulae:

$$
T\left(b_{0}, b\right)=T_{B}\left(b_{0}\right), N\left(b_{0}, b\right)=N_{B}\left(b_{0}\right)+\mu N_{B}(b)+\bar{\mu} N_{B}(\tau(b))-T_{B}\left(b_{0} b u \tau(b)\right) .
$$

From this, one gets an expression for the trace bilinear form on $J(B, \tau, u, \mu)$, defined by $T(x, y)=T(x y), x, y \in A$. Therefore, we have, for $x=\left(a_{0}, a\right), y=\left(b_{0}, b\right)$,

$$
T(x, y)=T_{B}\left(a_{0} b_{0}\right)+T_{B}(a u \tau(b))+T_{B}(b u \tau(a)) .
$$

The Albert algebra $A=J(B, \tau, u, \mu)$ is a division algebra if and only if the norm form is anisotropic over $k$.

The adjoint map: Let $A=J(B, \tau, u, \mu)$ and $x=\left(a_{0}, a\right)$. In this case, the adjoint map is given by

$$
x^{\#}=\left(a_{0}^{\#}-a u \tau(a), \bar{\mu} \tau(a)^{\#} u^{-1}-a_{0} a\right),
$$

where for $y \in B, 2 y^{\#}=y \times y$ as defined above. One has $N(x)=x x^{\#}=x^{\#} x$ for all $x \in A$.

Remarks. It is known that all Albert algebras arise from these two constructions (see [10]) and these are not mutually exclusive: there are Albert algebras of mixed type and others are of pure type. For a simple recipe for pure second constructions, see [26], and for examples of pure first constructions, see [13. Note that if $A$ is a pure first construction Albert division algebra, then every 9-dimensional subalgebra of $A$ must necessarily be of the form $D_{+}$for a degree 3 central division algebra $D$ over $k$. There is a cohomological characterization of pure second construction Albert algebras. An Albert algebra $A$ is a pure second construction if and only if $f_{3}(A) \neq 0$ (see 10, 40.5). However, such a characterization for pure first construction Albert algebras does not seem to be available in the literature. It is well known that any cubic subfield of an Albert division algebra reduces it; i.e., if $L \subset A$ is a cubic subfield, where $A$ is a division algebra over $k$, then $A \otimes L$ is reduced over $L$. Moreover, when $A$ is a first construction, every cubic subfield is a splitting field for $A$ (see [14]). It is known that an Albert division algebra $A$ over $k$ remains division (in particular, $\operatorname{Aut}(A)$ remains anisotropic) over any extension of $k$ of degree coprime to 3 (see [10]).

Pure first Tits construction Albert algebras: Albert algebras which arise through the first Tits construction and cannot arise from the second construction are called pure first constructions. Let $A$ be an Albert algebra over $k$ and let $S \subset A$ be a 9 -dimensional subalgebra. It is known that $S=D_{+}$or $S=\mathcal{H}(B, \tau)$ for $D$ a degree 3 central simple algebra over $k$ or $B$ a degree 3 central simple algebra over a quadratic extension $K / k$ with a unitary involution $\tau$ (see [18]). If $A$ is a pure first construction, then every 9-dimensional subalgebra $S$ must be of the form $D_{+}$, by remarks made before on 9-dimensional subalgebras of Albert algebras. Let $k_{0}$ be an algebraically closed field of characteristic not 2 or 3 and $n$ a positive integer. Then, by the work of Petersson and Racine ([13]), every Albert division algebra over the iterated Laurent series field $k=k_{0}\left(\left(x_{1}, \ldots, x_{n}\right)\right), n \geq 3$, is a pure first construction. 
The following characterisation of pure first Tits construction Albert division algebras was communicated to us by Holger P. Petersson:

Theorem 2.1. For an Albert division algebra over a field $k$ of arbitrary characteristic, the following conditions are equivalent.

(i) A is a pure first Tits construction.

(ii) $A$ is a first Tits construction and every separable cubic subfield of $A$ is cyclic.

(iii) Every separable cubic subfield of any isotope of $A$ is cyclic.

Proof. (i) implies (ii). Suppose $A$ is a pure first Tits construction and let $L \subset A$ be a separable cubic subfield. By [14, Cor. 4.5], there exists $\lambda \in k^{*}$ such that the inclusion $L \hookrightarrow A$ extends to an embedding from the first Tits construction $J=J(L, \lambda)$ to $A$. By the hypothesis, $J$ has the form $D_{+}$for some central associative division algebra $D$ of degree 3 over $k$. But by (Prop. 5.1, [18]), this forces the cubic extension $L / k$ to be cyclic.

(ii) implies (iii). If $A$ is a first Tits construction, then by ([14, Cor. 4.9) all its isotopes are isomorphic. Hence (iii) is a trivial consequence of (ii).

(iii) implies (i). Arguing indirectly, let us suppose that (iii) holds, but $A$ is not a pure first Tits construction. Then there exists a central associative division algebra $(B, \tau)$ of degree 3 with an involution of second kind over $k$ such that $\mathcal{H}(B, \tau)$ is a subalgebra of $A$ and $K$, the centre of $B$, is a separable quadratic field extension of $k$. Passing to an appropriate isotope of $\mathcal{H}(B, \tau)$, equivalently, replacing $\tau$ by $\operatorname{Int}(u) \circ \tau$ for some invertible $u \in \mathcal{H}(B, \tau)$, we may assume that the involution $\tau$ is distinguished (see [12, Theorem 2.10). But then, by loc.cit. Theorem 3.1, $\mathcal{H}(B, \tau)$, hence $A$, contains a separable cubic subfield with discriminant algebra $K / k$, which therefore cannot be cyclic.

The structure group of Albert algebras: Recall that every Albert algebra comes equipped with a cubic form $N$, called the norm. The isometries of $N$ form the $k$-rational points of a simply connected $k$-algebraic group of type $E_{6}$. This group contains the algebraic group $\operatorname{Aut}(A)$ of automorphisms of $A$; we denote its group of $k$-rational points by $A u t(A)$. The group of similarities of $N$ is called the structure group of $A$ and we denote it by $\operatorname{Str}(A)$. This coincides with the group of $k$-rational points of a strict inner $k$-form of $E_{6}$, which we denote by $\operatorname{Str}(A)$. Let $a \in A$ and $R_{a}$ denote the right multiplication by $a$ acting on $A$. We let $U_{a}=2 R_{a}^{2}-R_{a^{2}}$. Then $U_{a} \in \operatorname{Str}(A)$ for all invertible $a \in A$. The (normal) subgroup generated by $U_{a}, a$ invertible, is called the Inner structure group of $A$ and denoted by $\operatorname{Instr}(A)$; this also is the group of $k$-points of a certain algebraic group $\operatorname{Instr}(\mathbf{A})$. The group $\operatorname{Aut}(A) \cap \operatorname{Instr}(A)$ is called the group of inner automorphisms of $A$. Finally, recall that a norm isometry is an automorphism if and only if it fixes the identity element of $A$ (see [6], Section 7, Chapter VI).

\section{Moufang hexagons of type $27 / F$}

In this section, we explain the construction of Moufang hexagons of type $27 / F$ and connect them to the Kneser-Tits problem as well as the Tits-Weiss conjecture mentioned in the introduction. We reproduce below some of the material from [25], suited for this exposition. Let $A$ be an Albert division algebra over $k$ with norm map $N$ and trace map $T$. Let $U_{1}, U_{3}, U_{5}$ be three groups isomorphic to the additive group $(A,+)$ and let $U_{2}, U_{4}, U_{6}$ be three groups isomorphic to the additive group 
$(k,+)$. Let $x_{i}$ denote the isomorphism of $(A,+)$ or $(k,+)$ with $U_{i}$. We define a group $U_{+}$, generated by the $U_{i}$ subject to the commutation relations as follows (see [31], 8.13):

$$
\begin{gathered}
{\left[U_{1}, U_{2}\right]=\left[U_{2}, U_{3}\right]=\left[U_{3}, U_{4}\right]=\left[U_{4}, U_{5}\right]=\left[U_{5}, U_{6}\right]=1,} \\
{\left[U_{2}, U_{4}\right]=\left[U_{4}, U_{6}\right]=1,} \\
{\left[U_{1}, U_{4}\right]=\left[U_{2}, U_{5}\right]=\left[U_{3}, U_{6}\right]=1,} \\
{\left[x_{1}(a), x_{3}(b)\right]=x_{2}(T(a, b)),} \\
{\left[x_{3}(a), x_{5}(b)\right]=x_{4}(T(a, b)),} \\
{\left[x_{1}(a), x_{5}(b)\right]=x_{2}\left(-T\left(a^{\#}, b\right)\right) x_{3}(a \times b) x_{4}\left(T\left(a, b^{\#}\right)\right),} \\
{\left[x_{2}(t), x_{6}(u)\right]=x_{4}(t u),} \\
{\left[x_{1}(a), x_{6}(t)\right]=x_{2}(-t N(a)) x_{3}\left(t a^{\#}\right) x_{4}\left(t^{2} N(a)\right) x_{5}(-t a),}
\end{gathered}
$$

for all $a, b \in A$ and all $t, u \in k$. Here, $[x, y]$ denotes the commutator $x y x^{-1} y^{-1}$ and $\left[U_{i}, U_{j}\right]=1$ means $U_{i}$ centralizes $U_{j}$. We construct a (bipartite) graph $\Gamma$ from this data as follows: Let $\phi$ be a map from $\{1,2, \ldots, 6\}$ to the set of subgroups of $U_{+}$ defined by

$$
\phi(i)=U_{[1, i]}, \quad 1 \leq i \leq 3, \phi(i)=U_{[i-3,3]}, 4 \leq i \leq 6,
$$

where

$$
U_{[i, j]}=\left\langle U_{i}, U_{i+1}, \ldots, U_{j}\right\rangle, i \leq j<i+n ; U_{[i, j]}=1 \text { otherwise. }
$$

Let the vertex set of $\Gamma$ be defined by

$$
V(\Gamma)=\left\{(i, \phi(i) g) \mid 1 \leq i \leq 6, g \in U_{+}\right\},
$$

where $\phi(i) g$ is the right coset of $\phi(i)$ containing $g$. The edge set of $\Gamma$ is defined by

$$
E(\Gamma)=\{((i, R),(j, S))|| i-j \mid=1, R \cap S \neq \emptyset\} ;
$$

here $|i-j|$ is computed modulo 6. This gives a graph $\Gamma=(V(\Gamma), E(\Gamma))$, which is completely determined (up to isomorphism) by the 7 -tuple $\left(U_{+}, U_{1}, \ldots, U_{6}\right)$. We call the subgroups $U_{i}, 1 \leq i \leq 6$ the root groups of $\Gamma$. The graph $\Gamma$ is the Tits building associated to the $k$-algebraic group with index $E_{8,2}^{78}$ and anisotropic kernel the strict inner $k$-form of $E_{6}$ corresponding to the structure group of $A$. This graph is called a Moufang hexagon of type $27 / F$, where $F=k$ if $A$ is a first construction and a Moufang hexagon of type $K / k$ if $K / k$ is a separable quadratic extension of $k$ and $A$ is a second construction $J(B, \sigma, u, \mu)$ Albert division algebra, $B$ a degree 3 central division algebra with a unitary involution $\sigma$ over $K / k$.

Let $G$ be the group of type-preserving automorphisms of $\Gamma$ and let $G^{\dagger}$ denote the subgroup of $G$ generated by the root groups $U_{i}, 1 \leq i \leq 6$ of $\Gamma$ (see 31 for the definition). Then, by $(37.8,31]$,

$$
G / G^{\dagger} \simeq H / H^{\dagger}
$$

where $H$ is the pointwise stabilizer in $G$ of a 12-circuit in $\Gamma$ and $H^{\dagger}=G^{\dagger} \cap H$.

In (37.41, 31] ) the following result is proved: there is a canonical map from $H$ to $A u t(k)$. Let $H_{0}$ be the kernel of this map. Then $H^{\dagger} \subset H_{0}$. Let $G_{0}$ be the subgroup of $G$ containing $G^{\dagger}$ such that

$$
G_{0} / G^{\dagger}=H_{0} / H^{\dagger}
$$

Then $G_{0}$ is the group of $k$-rational points $\mathbf{G}(k)$ for an algebraic group defined over $k$ with Tits index $E_{8,2}^{78}$ as described in the introduction. Following the notation in 
[31, let $X_{1}$ denote the structure group of $A\left(\operatorname{Str}(A)\right.$ in our notation) and let $X_{1}^{\dagger}$ be the subgroup of $X_{1}$ generated by the $U$-operators $U_{a}, a \in A^{*}$ and the scalar multiplications $x \mapsto t x, t \in k^{*}$ (note that $X_{1}^{\dagger}=C$.Instr $(A)$ in our notation). Then it is shown in $(37.41,31])$ that

$$
H_{0} / H^{\dagger} \simeq X_{1} / X_{1}^{\dagger}
$$

By $([31], 42.3 .6)$, the root groups of $\Gamma$ are precisely the groups $\mathcal{U}_{\alpha}(k)$, where $\alpha$ is a nondivisible root corresponding to a maximal split $k$-torus $S$ in $\mathbf{G}$ and $\mathcal{U}_{\alpha}$ is the unipotent $k$-group corresponding to $\alpha$. Also,

$$
\mathbf{G}(k) / \mathbf{U}(k)=G_{0} / G^{\dagger}=H_{0} / H^{\dagger} \simeq X_{1} / X_{1}^{\dagger}=\operatorname{Str}(A) / C . \operatorname{Instr}(A),
$$

where $\mathbf{U}(k)$ denotes the subgroup of $\mathbf{G}(k)=G_{0}$ generated by the $k$-rational points of the unipotent radicals of parabolic $k$-subgroups of $\mathbf{G}$. The Kneser-Tits problem is to determine if the quotient $\mathbf{G}(k) / \mathbf{U}(k)$ is trivial. The Tits-Weiss conjecture asserts that the quotient $G_{0} / G^{\dagger} \simeq S \operatorname{tr}(A) / C$.Instr $(A)$ is trivial.

\section{Automorphisms of Albert algebras}

In this section, we prove some results about automorphisms of Albert algebras in general. Some of these are intended for the proof of the Tits-Weiss conjecture, but others may be of independent interest. Since some of the crucial results need characteristic restrictions, we assume at the outset that the characteristic of the base field $k$ is different from 2 and 3. Let $A$ be an Albert algebra over $k$ and let $A_{0}$ denote the subspace of elements of $A$ having zero trace. We shall denote the algebraic group of automorphisms of $A$ by $\operatorname{Aut}(A)$ and $A u t(A)$ will denote its group of $k$-rational points. Also, for a subalgebra $S \subset A, G_{S}=\operatorname{Aut}(A / S)$ will denote the algebraic subgroup of $\operatorname{Aut}(A)$ consisting of automorphisms fixing $S$ pointwise. For a central simple algebra $D$ over $k$, we will denote by $\mathbf{S L}(1, D)$ the algebraic group of norm 1 elements in $D$, and $S L(1, D)$ will denote its group of $k$ rational points. We need descriptions of certain subgroups of $\operatorname{Aut}(A)$ for our purpose; we include it here (see [10], Chapter IX, Section 39). We have

Proposition 4.1. Let $A=\mathcal{H}_{3}(C, \Gamma)$ be a reduced Albert algebra over $k$ and let $H \subset \boldsymbol{A u t}(A)$ be the algebraic subgroup of $\operatorname{Aut}(A)$ consisting of all automorphisms of $A$ which fix the three diagonal idempotents in $A$. Then $H$ is a $k$-subgroup of type $D_{4}$; in fact $H \simeq \operatorname{Spin}\left(n_{C}\right)$, where $n_{C}$ is the norm on the octonion algebra $C$.

We will denote the subgroup in the proposition by $\mathbf{S p i n}(8)$ when the norm form $n_{C}$ of $C$ is not important in the discussion.

Corollary 4.1. Let $A$ be an Albert algebra over $k$ and $L \subset A$ be a cubic étale subalgebra of $A$. Then $\operatorname{Aut}(A / L)$ is a k-subgroup of $\operatorname{Aut}(A)$ of type $D_{4}$.

Proposition 4.2. Let $A$ be an Albert algebra arising from the first construction. Let $D_{+} \subset A$. Then, with the notation introduced above, $\operatorname{Aut}\left(A / D_{+}\right)$is a k-subgroup of $\boldsymbol{A u t}(A)$, isomorphic to $\mathbf{S L}(1, D)$ over $k$.

We now prove our first key result. The proof rests on the conjugacy theorem for maximal tori in algebraic groups. Recall that for an Albert algebra $A$ the subspace of trace zero elements is denoted by $A_{0}$.

Theorem 4.1. Let $\phi \in A u t(A)$. There exists a nonzero element $x_{0} \in A_{0}$ such that $\phi\left(x_{0}\right)=x_{0}$. 
Proof. Since $\phi$ is an automorphism, we have $\phi\left(A_{0}\right)=A_{0}$. To prove the assertion, it suffices to prove that 1 is an eigenvalue for the restriction of $\phi$ to $A_{0}$. To prove the assertion on eigenvalues of $\phi$, we may assume $k$ is algebraically closed and, by considering the Jordan decomposition of $\phi$, assume that $\phi$ is semisimple. We can write $A=\mathcal{H}_{3}(\mathcal{C}, 1)$, for the split Cayley algebra $\mathcal{C}$ over $k$. Let $T \subset G=\operatorname{Aut}(A)$ be a maximal torus containing $\phi$. By Proposition 4.1, the subgroup $H$ of $G$, consisting of all automorphisms of $A$ that fix the three diagonal idempotents in $A$, is isomorphic to $\operatorname{Spin}(8)$ and hence has rank 4. Therefore, for some $g \in G, g^{-1} T g \subset H$. Hence $g^{-1} \phi g$ fixes the three diagonal idempotents. This implies that $g^{-1} \phi g$ fixes $a=$ $\operatorname{diag}(1,-1,0) \in A_{0}$. Hence $\phi$ fixes $x_{0}=g(a) \in A_{0}$.

Corollary 4.2. Let $A$ be an Albert division algebra over $k$ and $\phi \in A u t(A)$ an automorphism of $A$. Then $\phi$ fixes a cubic subfield of $A$ pointwise.

Proof. By the above theorem, there is a nonzero element $x_{0} \in A_{0}$ such that $\phi\left(x_{0}\right)=$ $x_{0}$. Let $L$ be the subalgebra generated by $x_{0}$. Then $L$ is a cubic field extension of $k$ and clearly $\phi$ fixes $L$ pointwise.

Theorem 4.2. Let $A$ be an Albert algebra over $k$, char $(k) \neq 2,3$, and suppose $\phi \in \operatorname{Aut}(A)$ is semisimple. Then $\phi$ fixes a cubic étale subalgebra $L \subset A$ pointwise.

Proof. Let $B=A^{\phi}$ denote the subalgebra of fixed points of $\phi$ in $A$. Then $B$ is compatible with arbitrary base field extensions; i.e. for any extension $M / k,\left(A \otimes_{k}\right.$ $M)^{\phi \otimes 1}=A^{\phi} \otimes_{k} M$. By an argument as in the proof of the above theorem, $A^{\phi} \otimes_{k} \bar{k}$ contains a split cubic étale subalgebra, where $\bar{k}$ is a fixed algebraic closure of $k$. In particular, the nil radical of $A^{\phi} \otimes_{k} \bar{k}$ has codimension at least 3 over $\bar{k}$. By [16], $N=$ Nil radical of $A^{\phi}$ coincides with the radical of the bilinear trace of $A^{\phi}$. Hence $\bar{N}=N \otimes_{k} \bar{k}=\operatorname{Nilrad}\left(A^{\phi} \otimes_{k} \bar{k}\right)$. Since $\operatorname{char}(k) \neq 2,3$, the algebra $A^{\phi} / N$ is separable. Hence there exists a cubic separable Jordan subalgebra $S \subset A^{\phi}$ such that $A^{\phi}=S \oplus N$, a direct sum of subspaces. But $\operatorname{dim}(S) \geq 3$; hence $S$ contains a cubic étale subalgebra $L$ and since $S \subset A^{\phi}, \phi$ fixes $L$ pointwise.

Remark. The original proof of this theorem had some mistakes. The proof above was suggested to us by Holger P. Petersson.

Corollary 4.3. Let $G$ be a group of type $F_{4}$, defined and anisotropic over a (perfect) field $k$, $\operatorname{char}(k) \neq 2,3$. Let $T$ be a maximal torus in $G$, defined over $k$. Then there exists a simply connected subgroup $H \subset G$ defined over $k$, of type $D_{4}$, such that $T \subset H$ over $k$.

Proof. First assume that $k$ is an algebraic extension of a finite field. Then $\operatorname{Br}(k)=0$ (see e.g. [22]). Hence any Albert algebra over $k$ is split and every group of type $F_{4}$ over $k$ is split. Hence $k$ is not an algebraic extension of a finite field and we can apply [1, Proposition 8.8] to find a regular element $\phi \in T(k)$. Let $A$ be the Albert algebra over $k$ such that $G=\operatorname{Aut}(A)$. Since $G$ is anisotropic over $k$ and $k$ is perfect, by [21, Proposition 6.3], all elements of $\operatorname{Aut}(A)(k)=\operatorname{Aut}(A)$ are semisimple. Hence by the theorem above, there is a cubic étale subalgebra $L \subset A$ fixed pointwise by $\phi$. Let $G_{L}=\operatorname{Aut}(A / L)$ be the subgroup of $G$ consisting of automorphisms of $A$ that fix $L$ pointwise. Then $G_{L}$ is a simply connected group of type $D_{4}$, defined over $k$ ([10, Chapter IX). Clearly $\phi \in G_{L}$. Let $T^{\prime} \subset G_{L}$ be a maximal torus defined over $k$ such that $\phi \in T^{\prime}$. But then $T^{\prime}$ is also maximal in 
$G$. Since $\phi$ is regular, it belongs to a unique maximal torus. Hence $T=T^{\prime} \subset G_{L}$. Hence we have proved the assertion.

Some automorphisms of first Tits constructions: We recall some known automorphisms of Albert algebras which are Tits first constructions and introduce notation for them for our future use. The facts used on Albert algebras can be found in Chapter IX of [10] or Chapter IX of [6].

Let $A=J(D, \mu)$ be an Albert algebra and $a, b \in D^{*}$ be such that $N_{D}(a)=$ $N_{D}(b)$. Let $\psi_{a, b}: A \longrightarrow A$ be the automorphism of $A$ given by

$$
\psi_{a, b}(x, y, z)=\left(a x a^{-1}, a y b^{-1}, b z a^{-1}\right) .
$$

Then clearly $\psi_{a, b}\left(D_{0}\right)=D_{0}$ and it is known (see 10, Chapter IX) that any automorphism of $A$ stabilizing $D_{0}$ is of this form. A special case of this merits a separate mention. Let $p \in S L(1, D)$. Let $\mathcal{J}_{p}: A \longrightarrow A$ denote the automorphism $\psi_{1, p^{-1}}$, i.e.

$$
\mathcal{J}_{p}(x, y, z)=\left(x, y p, p^{-1} z\right) .
$$

We shall write $\mathcal{I}_{a}$ for the automorphism $\mathcal{I}_{a}(x, y, z)=\left(a x a^{-1}, a y a^{-1}, a z a^{-1}\right)$. Hence $\mathcal{I}_{a}=\psi_{a, a}$. Observe that

$$
\psi_{a, b} \psi_{c, d}=\psi_{a c, b d}, \mathcal{I}_{a b}=\mathcal{I}_{a} \mathcal{I}_{b}, \mathcal{J}_{a b}=\mathcal{J}_{b} \mathcal{J}_{a}, a, b \in D^{*} .
$$

It is known that any automorphism of $A$ fixing $D_{0}$ pointwise is of this form. It is also known that the subgroup $\operatorname{Aut}\left(A / D_{+}\right)$of $\operatorname{Aut}(A)$ fixing $D_{+}$pointwise is isomorphic to $\mathbf{S L}(1, D)$ (see [10], Section 39.B). We record the extension of automorphisms of subalgebras of the form $D_{+}$of $A$ below, since we will use this frequently in the paper (see [10, 39.B):

Lemma 4.1. Let $A$ be an Albert algebra over $k$ and $D_{+} \subset A$. Then any automorphism of $D$ extends to an automorphism of $A$. Since every automorphism of $D$ is inner, we may extend any automorphism of $D$ to an automorphism by $\psi_{a, b}$, for suitable $a, b \in D^{*}$ and, in particular, $\mathcal{I}_{a}=\psi_{a, a}$ gives such an extension for a suitable $a \in D^{*}$. If $A=J(D, \mu)$, then every automorphism of $A$ stabilizing $D_{0}$ is of the form $\psi_{a, b}$ for suitable $a, b \in D^{*}$.

Automorphisms of second Tits constructions: Let $A$ be an Albert algebra over a field $k$. We will briefly discuss here automorphisms of $A$ that leave a 9-dimensional subalgebra invariant. Let $A=J(B, \sigma, u, \mu)$ be a second Tits construction Albert division algebra. Let $\sigma^{\prime}=\operatorname{Int}(u) \circ \sigma$. We then have the special unitary group of $\left(B, \sigma^{\prime}\right)$ given by

$$
S U\left(B, \sigma^{\prime}\right)=\left\{x \in B \mid x \sigma^{\prime}(x)=1, N_{B}(x)=1\right\} .
$$

Let $p \in S U(B, \sigma)$ and $q \in S U\left(B, \sigma^{\prime}\right)$. Let $\phi_{p, q}$ denote the automorphism of $A$ given by

$$
\phi_{p, q}(a, b)=(p a \sigma(p), p b q) .
$$

Then $\phi_{p, q}$ stabilizes $\mathcal{H}(B, \sigma)$ and it is known (see [10], 39.16) that any automorphism of $A$ stabilizing $\mathcal{H}(B, \sigma)$ is of this form. Let $p \in S U(B, \sigma)$. We will denote $\phi_{p, 1}$ simply by $\phi_{p}$.

Fixed points of subgroups of $\operatorname{Aut}(A)$ : Here we discuss the subalgebra of fixed points of certain subgroups of $G=\operatorname{Aut}(A)$. For a subgroup $H \subset G$, we denote the subalgebra of $A$ of fixed points of $H$ by

$$
A^{H}=\{x \in A \mid h(x)=x(h \in H)\} .
$$


Theorem 4.3. Let $A$ be an Albert algebra over a field $k$, char $(k) \neq 2,3$. Let $T \subset G=\operatorname{Aut}(A)$ be a maximal torus defined over $k$. Then $A^{T}$ is a cubic étale subalgebra of $A$.

We need the following lemma for the proof.

Lemma 4.2. Let $\mathcal{C}$ be a split Cayley algebra over $k$ and let $A=\mathcal{H}_{3}(\mathcal{C}, 1)$. Then there exists a semisimple automorphism $\phi \in A u t(A)$ such that $\operatorname{Dim}_{k}\left(A^{\phi}\right)=3$.

Proof. Write $A$ as a first construction, $A=J\left(M_{3}(k), 1\right)$. Choose $\alpha, \beta, \gamma \in k^{*}$, each different from 1, pairwise distinct, such that $\alpha \beta \gamma=1$, and $a=\operatorname{Diag}(\alpha, \beta, \gamma) \neq \pm 1$. Define $\psi: A \longrightarrow A$ by $\psi(x, y, z)=\left(a x a^{-1}, a y, z a^{-1}\right)$. By the choice of the matrix $a$, it is clear that $\psi$ is semisimple. We have

$$
\psi(x, y, z)=(x, y, z) \Longleftrightarrow\left(a x a^{-1}, a y, z a^{-1}\right)=(x, y, z)
$$

$\Longleftrightarrow x$ centralizes $a$ and $y=z=0$ since $a \neq \pm 1$. Hence $x \in k \times k \times k$ and $A^{\psi}=(k \times k \times k, 0,0)$. Therefore $\operatorname{Dim}_{k}\left(A^{\psi}\right)=3$.

Proof of theorem. Let $\phi \in A u t\left(A \otimes_{k} \bar{k}\right)$ be a semisimple automorphism such that $\operatorname{Dim}_{\bar{k}}\left(A \otimes_{k} \bar{k}\right)=3$, which exists by the lemma. Let $T^{\prime} \subset G$ be a maximal torus with $\phi \in T^{\prime}$. For some $g \in G$ we have $g T g^{-1}=T^{\prime}$. Hence $\operatorname{Dim}_{k}\left(A^{T}\right) \leq 3$. We now apply Theorem 4.2 to a regular element in $T(k)$ to conclude $\operatorname{Dim}_{k}\left(A^{T}\right) \geq 3$. Hence $\operatorname{Dim}_{k}\left(A^{T}\right)=3$ and that $A^{T}$ is étale follows from Corollary 4.2 .

Corollary 4.4. Let $S \subset \operatorname{Aut}(A)$ be a k-torus. Then $\operatorname{Dim}\left(A^{S}\right) \geq 3$.

Proof. Let $S \subset T$, where $T \subset \operatorname{Aut}(A)$ is a $k$-maximal torus. It follows that $A^{T} \subset A^{S}$ and the result follows from the above theorem.

Corollary 4.5. Let $L \subset A$ be a cubic étale subalgebra. Then for $G_{L}=\operatorname{Aut}(A / L)$, $A^{G_{L}}=L$.

Proof. We have, for a maximal $k$-torus $T \subset G_{L}, \operatorname{Dim}(T)=4$. Hence $T$ is maximal $\operatorname{in} \operatorname{Aut}(A)$ as well and hence we have

$$
L \subset A^{G_{L}} \subset A^{T}=L .
$$

Examples. (i) Let $A=J(D, \mu)$ be an Albert algebra with $D$ a degree 3 division algebra. Let $L \subset D$ be a cubic cyclic subfield. Let $p \in L$ with $N_{L / k}(p)=1$. Then the automorphism $\mathcal{I}_{p}: A \rightarrow A,(x, y, z) \mapsto\left(x, y p, p^{-1} z\right) \in \operatorname{Aut}\left(A / D_{0}\right)$. The subgroup $S=\left\{\mathcal{I}_{p} \mid p \in L, N_{L / k}(p)=1\right\}$ is a torus in $\mathbf{S L}(1, D)$; in fact, $S=R_{L / k}^{(1)}\left(\mathbb{G}_{m}\right)$. Hence $S$ is a torus with $\operatorname{Dim}(S)=2$. If $(x, y, z) \in A^{S}$, then we have $\left(x, y p, p^{-1} z\right)=(x, y, z)$ for all $p \in S$. This implies in particular, $y p=y, p^{-1} z=z$ for all $p \in S$. Hence $y=z=0$. Therefore $A^{S}=D_{0}$ and $\operatorname{Dim}\left(A^{S}\right)=9$.

(ii) Consider again $A=J(D, \mu)$ with $D$ and $L$ as above. Let $p \in L$ with $N_{L / k}(p)=1$. Consider the automorphism $\psi_{p, 1}: A \rightarrow A$ given by $(x, y, z) \mapsto$ $\left(p x p^{-1}, p y, z p^{-1}\right)$. Then the subgroup $S=\left\{\psi_{p, 1} \mid p \in L, N_{L / k}(p)=1\right\}$ is a torus in $\operatorname{Aut}(A), \operatorname{Dim}(S)=2$. Now if $(x, y, z) \in A^{S}$, then $\left(p x p^{-1}, p y, z p^{-1}\right)=(x, y, z)$ for all $p \in L, N_{L / k}(p)=1$. Hence $y=z=0$ and $x \in L$ because $L \subset D$ is a maximal subfield of $D$. Therefore $A^{S}=L$ and $\operatorname{Dim}\left(A^{S}\right)=3$. This, together with Example (i), shows that the dimension of the fixed point subalgebra of a torus does not determine the dimension of the torus. 


\section{Tits-Weiss CONJECture}

In this section, we take up the proof of the conjecture of Tits and Weiss on the structure group of Albert division algebras over a field. We continue with the restrictions on the characteristic of the base field. We will prove results on automorphisms and norm similarities of Albert division algebras. When the result holds for pure first constructions, we emphasize this by highlighting this adjective in boldface. We begin with recalling some classical results on automorphisms of Cayley algebras. We will reprove a theorem of Jacobson ([7]), which immediately generalizes in the context of Albert algebras.

The case of Cayley algebras: Let $\mathcal{C}$ be a Cayley algebra over a field $k$. Let $a \in \mathcal{C}$. Let $a_{L}, a_{R}$ respectively denote the left and the right multiplication maps on $\mathcal{C}$ for $a \in \mathcal{C}$. Then the flexible law in $\mathcal{C}$ says that, for $x \in \mathcal{C}, a(x a)=(a x) a$. Hence $a_{L} a_{R}=a_{R} a_{L}$. Let $U_{a}=a_{L} a_{R}$. An automorphism $\eta$ of $\mathcal{C}$ is said to be inner if $\eta=U_{a_{1}} \cdots U_{a_{r}}$ for some $a_{1}, \ldots, a_{r} \in \mathcal{C}$. We call a nontrivial automorphism $\tau$ of $\mathcal{C}$ a reflection if $\tau^{2}=1$ and if the fixed point subalgebra $\mathcal{B}$ of $\tau$ is 4 -dimensional (hence is a quaternion subalgebra). We can decompose $\mathcal{C}$ as $\mathcal{B} \oplus \mathcal{B}^{\perp}$. Then $\tau$ is identity on $\mathcal{B}$ and -1 on $\mathcal{B}^{\perp}$. We have the following two theorems of Jacobson ([7]). We will give a different proof of the second theorem, only for Cayley division algebras, since that generalizes to the Albert algebra case.

Theorem 5.1. Every reflection in a Cayley algebra is inner.

Theorem 5.2. Every automorphism of $\mathcal{C}$ is a product of reflections. In particular, every automorphism of $\mathcal{C}$ is inner.

Proof. We will prove the assertion when $\mathcal{C}$ is a division algebra. So let us assume $\mathcal{C}$ is a division algebra and $\eta$ is an automorphism of $\mathcal{C}$. Let $\mathcal{C}_{0}$ denote the subspace of elements of $\mathcal{C}$ of trace 0. Then $\eta$ maps $\mathcal{C}_{0}$ to itself. By a theorem of Cartan and Dieudonné, there is $x_{0} \neq 0 \in \mathcal{C}_{0}$ such that $\eta\left(x_{0}\right)=x_{0}$. Let $K=k\left(x_{0}\right)$ be the subalgebra of $\mathcal{C}$ generated by $x_{0}$. Then $K / k$ is a quadratic field extension. Let $\sigma: K \longrightarrow K$ be the nontrivial $k$-automorphism of $K$. Let $\mathcal{H} \subset \mathcal{C}$ be a quaternion subalgebra containing $K$. By a Skolem-Noether type theorem for composition algebras (see [10], 33.21), $\sigma$ extends to an automorphism $\mathcal{H} \longrightarrow \mathcal{H}$, which in turn extends to an automorphism $\widetilde{\sigma}: \mathcal{C} \longrightarrow \mathcal{C}$. Hence $\widetilde{\sigma}$ is an automorphism of $\mathcal{C}$ that stabilizes a quaternion subalgebra and hence is a product of reflections by a theorem of $\mathrm{M}$. Wonenburger ([36]). Consider the automorphism $\psi=\eta \widetilde{\sigma}^{-1}$ of $\mathcal{C}$. Then $\psi$ does not fix $K$ pointwise, since $\widetilde{\sigma}$ does not. Let $L$ be a quadratic extension of $k$ contained in $\mathcal{C}$ that is fixed pointwise by $\psi$. Then $\psi(K)=K$ and $\psi(L)=L$. Hence $\psi$ stabilizes the quaternion subalgebra $\mathcal{B}$ generated by $K$ and $L$. By the aforementioned theorem of $\mathrm{M}$. Wonenburger $([36]), \psi$ is a product of reflections. Hence $\eta$ itself is a product of reflections.

Back to Albert algebras: We will now adopt a similar approach for Albert division algebras. To begin with, we need to know if every automorphism of an Albert algebra fixes a nonzero element of trace zero. This has already been settled in Theorem 4.1. We have shown that every automorphism of an Albert division algebra $A$ fixes a cubic subfield of $A$.

Definition. We define an automorphism of an Albert division algebra to be cyclic if it fixes a cyclic cubic subfield of $A$. 
Theorem 5.3. Let $A$ be a first Tits construction Albert division algebra over $k$. Let $G=\operatorname{Aut}(A)$ and $\phi \in G$. Assume $\phi$ is cyclic. Then $\phi$ is a product of two automorphisms of $A$, each stabilizing a 9-dimensional subalgebra of $A$.

Proof. Let $L \subset A$ be a cubic cyclic subfield that is fixed pointwise by $\phi$. Let $D$ be a degree 3 central division algebra over $k$ such that $D_{+} \subset A$ and $L \subset D_{+}$ (see [14, Cor. 4.6). Then the inclusion $D_{+} \subset A$ extends to an isomorphism $\theta: A \longrightarrow J(D, \mu)$ for a suitable $\mu \in k^{*}$ such that $\theta\left(D_{+}\right)=D_{0}$. Let $\operatorname{Gal}(L / k)=\langle\sigma\rangle$. By the classical Skolem-Noether theorem, $\sigma: L \longrightarrow L$ extends to an automorphism $\widetilde{\sigma}: D \longrightarrow D$. Hence there exists $a \in D^{*}$ such that $\widetilde{\sigma}(x)=a x a^{-1}$ for all $x \in D$. This in turn extends to an automorphism $\widetilde{\sigma}_{D}: J(D, \mu) \longrightarrow J(D, \mu)$ by $\widetilde{\sigma}_{D}=\mathcal{I}_{a}$. Consider $\Psi=\phi \theta^{-1} \widetilde{\sigma}_{D} \theta: A \longrightarrow A$. Note that, for $x \in L, \theta^{-1} \widetilde{\sigma}_{D} \theta(x)=\sigma(x)$. Hence $\theta^{-1} \widetilde{\sigma}_{D} \theta \notin G_{L}$. Since $\phi \in G_{L}$, this means that $\Psi \notin G_{L}$. Let $M \subset A$ be a cubic field extension of $k$ such that $\Psi \in G_{M}$ (this is possible by Corollary 4.2). Then the subalgebra $S=\langle L, M\rangle$ is 9-dimensional (see [15], 2.10, [18]) and $\Psi(L)=L, \Psi(M)=M$. Hence $\Psi(S)=S$. But the automorphism $\Pi=\theta^{-1} \widetilde{\sigma}_{D} \theta$ maps the 9-dimensional subalgebra $\theta^{-1}\left(D_{0}\right)$ to itself. Now $\phi=\Psi \Pi^{-1}$ is a product of two automorphisms of the required type.

Corollary 5.1. Let $A$ be as in the theorem and $\phi \in \operatorname{Aut}(A)$ be such that $\phi(L)=L$ for a cubic cyclic extension $L \subset A$. Then $\phi$ is a product of three automorphisms, each stabilizing a 9-dimensional subalgebra of $A$.

Proof. Let $\operatorname{Gal}(L / k)=\langle\sigma\rangle$. Let $D_{+} \subset A$ be such that $L \subset D_{+}$and $\widetilde{\sigma_{D}} \in \operatorname{Aut}(A)$ be exactly as in the proof of the above theorem. Then $\phi^{-1} \widetilde{\sigma_{D}}$ fixes $L$ pointwise. Hence by the theorem, $\phi^{-1} \widetilde{\sigma_{D}}$ is a product of two automorphisms, each stabilizing a 9-dimensional subalgebra of $A$. Since $\widetilde{\sigma_{D}}$ leaves $D_{+}$stable, the claim follows.

Corollary 5.2. Let $A$ be a pure first Tits construction Albert division algebra. Then every automorphism of $A$ is a product of two automorphisms of $A$, each stabilizing a 9-dimensional subalgebra of $A$.

Proof. Let $A$ be a pure first construction Albert division algebra over $k$. Let $\phi \in$ Aut $(A)$. By Corollary 4.2, $\phi$ fixes a cubic subfield $L \subset A$. By Theorem 2.1, $L$ is a cyclic extension of $k$. Hence $\phi$ is cyclic and the result follows from the above theorem.

Our next goal is to prove that every automorphism of a pure first construction Albert division algebra that stabilizes a 9-dimensional subalgebra is inner. This will be carried out in two steps. We will first prove that every automorphism which stabilizes a 9-dimensional subalgebra of $A$ is a product of automorphisms fixing 9-dimensional subalgebras. We will then prove that automorphisms that fix 9-dimensional subalgebras pointwise are indeed inner. While the first assertion is easy to prove, the second one is delicate and needs a bit more care. We will prove better results for pure first constructions, while proving some general statements for first constructions. We begin with

Theorem 5.4. Let $A$ be an Albert division algebra which is a pure first construction. Let $\phi \in \operatorname{Aut}(A)$ be such that $\phi$ stabilizes a 9-dimensional subalgebra. Then $\phi$ is a product of automorphisms that fix 9-dimensional (separable) subalgebras.

Proof. Suppose $\phi \in A u t(A)$ stabilizes the subalgebra $D_{+} \subset A$. The inclusion $D_{+} \hookrightarrow A$ induces an isomorphism $\theta: A \longrightarrow J(D, \mu)$ for some $\mu \in k^{*}$ such that 
$\theta\left(D_{+}\right)=D_{0}$. Then $\psi=\theta \phi \theta^{-1}$ is an automorphism of $J(D, \mu)$ and $\psi$ stabilizes $\theta\left(D_{+}\right)=D_{0}$. By Lemma 4.1, we can find elements $a, b \in D^{*}$ such that $N_{D}(a)=$ $N_{D}(b)$ and $\psi$ is given by

$$
\psi(x, y, z)=\left(a x a^{-1}, a y b^{-1}, b z a^{-1}\right) .
$$

Recall that, for $a \in D^{*}, \mathcal{I}_{a}$ denotes the automorphism of $J(D, \mu)$ given by

$$
\mathcal{I}_{a}(x, y, z)=\left(a x a^{-1}, a y a^{-1}, a z a^{-1}\right)
$$

and, for $p \in S L(1, D), \mathcal{J}_{p}$ denotes the automorphism of $J(D, \mu)$ given by

$$
\mathcal{J}_{p}(x, y, z)=\left(x, y p, p^{-1} z\right) .
$$

We have

$$
\begin{aligned}
\mathcal{J}_{a b^{-1}} \mathcal{I}_{a}(x, y, z) & =\mathcal{J}_{a b^{-1}}\left(a x a^{-1}, a y a^{-1}, a z a^{-1}\right) \\
& =\left(a x a^{-1}, a y a^{-1} a b^{-1}, b a^{-1} a z a^{-1}\right) \\
& =\left(a x a^{-1}, a y b^{-1}, b z a^{-1}\right)=\psi(x, y, z) .
\end{aligned}
$$

Hence $\psi=\mathcal{J}_{a b^{-1}} \mathcal{I}_{a}$. We only have to check that $\mathcal{I}_{a}$ fixes some 9-dimensional separable subalgebra. But this is clear, since either $a \in k^{*}$, in which case $\mathcal{I}_{a}=1$ or $L=k(a)$ is a cubic extension of $k$ contained in $D$ and then $\mathcal{I}_{a}$ fixes the subalgebra $S=L \times L \times L$ pointwise. Now

$$
\phi=\theta^{-1} \psi \theta=\left(\theta^{-1} \mathcal{J}_{a b^{-1}} \theta\right)\left(\theta^{-1} \mathcal{I}_{a} \theta\right) .
$$

The automorphism $\theta^{-1} \mathcal{J}_{a b^{-1}} \theta$ fixes $\theta^{-1}\left(D_{0}\right) \subset A$ pointwise while the automorphism $\theta^{-1} \mathcal{I}_{a} \theta$ fixes the subalgebra $\theta^{-1}(L \times L \times L) \subset A$ pointwise.

Remark. Note that the above proof gives more than what is stated in the theorem. Given an Albert algebra $A$ over $k$, call a 9-dimensional subalgebra of the first (resp. of the second) kind if it is isomorphic to $D_{+}$(resp. to $\mathcal{H}(D, \tau)$ ), where $D$ is a central simple associative $k$-algebra of degree 3 (resp. a central simple associative algebra of degree 3 over some separable quadratic extension of $k$ with $\tau: D \rightarrow D$ a unitary involution). With this notation, we have the following assertion:

Let $A$ be an Albert division algebra over $k$. Then every automorphism of $A$ that stabilizes a 9-dimensional subalgebra of the first kind, is a product of two automorphisms, each of which fixes some 9-dimensional separable subalgebra of $A$.

In fact, the first factor of the product exhibited in the proof fixes a 9-dimensional subalgebra of the first kind, while the subalgebra fixed by the second factor is always separable and is of the first kind if and only if $L=k(a)$ is a cyclic cubic extension of $k$.

Some computations with $U$ operators (first construction): We refer to [15] for basic formulae on $U$-operators for first construction Albert algebras. Let $A=J(D, \mu)$ be a first construction Albert algebra over $k$. For $x=\left(x_{0}, x_{1}, x_{2}\right), y=$ $\left(y_{0}, y_{1}, y_{2}\right) \in A$, we define

$$
\begin{aligned}
x \times y= & \left(x_{0} \times y_{0}-x_{1} y_{2}-y_{1} x_{2}, \mu^{-1} x_{2} \times y_{2}-x_{0} y_{1}-y_{0} x_{1},\right. \\
& \left.\mu x_{1} \times y_{1}-x_{2} y_{0}-y_{2} x_{0}\right), \\
T(x, y)= & T_{D}\left(x_{0}, y_{0}\right)+T_{D}\left(x_{1}, y_{2}\right)+T_{D}\left(x_{2}, y_{1}\right)
\end{aligned}
$$


and

$$
x^{\#}=\left(x_{0}^{\#}-x_{1} x_{2}, \mu^{-1} x_{2}^{\#}-x_{0} x_{1}, \mu x_{1}^{\#}-x_{2} x_{0}\right) ;
$$

here $T_{D}(a, b)=T_{D}(a b)$ and $T_{D}$ is the reduced trace on $D$. The $U$-operators on $A$ are given by

$$
U_{x}(y)=T(x, y) x-x^{\#} \times y, x, y \in A .
$$

We have, using these formulae, for $a, b, c \in D$,

$$
(a, 0,0)^{\#}=\left(a^{\#}, 0,0\right),(0, b, 0)^{\#}=\left(0,0, \mu b^{\#}\right),(0,0, c)^{\#}=\left(0, \mu^{-1} c^{\#}, 0\right) .
$$

We have therefore,

$$
\begin{aligned}
& (a, 0,0)^{\#} \times(d, e, f)=\left(a^{\#}, 0,0\right) \times(d, e, f)=\left(a^{\#} \times d,-a^{\#} e,-f a^{\#}\right), \\
& (0, b, 0)^{\#} \times(d, e, f)=\left(0,0, \mu b^{\#}\right) \times(d, e, f)=\left(-\mu e b^{\#}, b^{\#} \times f,-\mu b^{\#} d\right), \\
& (0,0, c)^{\#} \times(d, e, f)=\left(0, \mu^{-1} c^{\#}, 0\right) \times(d, e, f)=\left(-\mu^{-1} c^{\#} f,-\mu^{-1} d c^{\#}, c^{\#} \times e\right) .
\end{aligned}
$$

We also note that for $x, y \in D, T_{D}(x y) x-x^{\#} \times y=x y x$. We have

$$
T((a, 0,0),(d, e, f))=T_{D}(a d) .
$$

Hence

$$
\begin{aligned}
U_{(a, 0,0)}(d, e, f) & =T_{D}(a d)(a, 0,0)-(a, 0,0)^{\#} \times(d, e, f) \\
& =\left(T_{D}(a d) a, 0,0\right)-\left(a^{\#} \times d,-a^{\#} e,-f a^{\#}\right) \\
& =\left(T_{D}(a d) a-a^{\#} \times d, a^{\#} e, f a^{\#}\right) \\
& =\left(a d a, a^{\#} e, f a^{\#}\right) .
\end{aligned}
$$

Again,

$$
T((0, b, 0)(d, e, f))=T_{D}(b f)
$$

and hence

$$
\begin{aligned}
U_{(0, b, 0)}(d, e, f) & =T_{D}(b f)(0, b, 0)-(0, b, 0)^{\#} \times(d, e, f) \\
& =\left(0, T_{D}(b f) b, 0\right)-\left(-\mu e b^{\#}, b^{\#} \times f,-\mu b^{\#} d\right) \\
& =\left(\mu e b^{\#}, T_{D}(b f) b-b^{\#} \times f, \mu b^{\#} d\right) \\
& =\left(\mu e b^{\#}, b f b, \mu b^{\#} d\right) .
\end{aligned}
$$

Finally,

$$
T((0,0, c),(d, e, f))=T_{D}(c e)
$$

hence

$$
\begin{aligned}
U_{(0,0, c)}(d, e, f) & =T_{D}(c e)(0,0, c)-(0,0, c)^{\#} \times(d, e, f) \\
& =\left(0,0, T_{D}(c e) c\right)-\left(-\mu^{-1} c^{\#} f,-\mu^{-1} d c^{\#}, c^{\#} \times e\right) \\
& =\left(\mu^{-1} c^{\#} f, \mu^{-1} d c^{\#}, T_{D}(c e) c-c^{\#} \times e\right) \\
& =\left(\mu^{-1} c^{\#} f, \mu^{-1} d c^{\#}, c e c\right) .
\end{aligned}
$$

We summarize the above computations as

$$
\begin{aligned}
& U_{(a, 0,0)}(d, e, f)=\left(a d a, a^{\#} e, f a^{\#}\right), \\
& U_{(0, b, 0)}(d, e, f)=\left(\mu e b^{\#}, b f b, \mu b^{\#} d\right), \\
& U_{(0,0, c)}(d, e, f)=\left(\mu^{-1} c^{\#} f, \mu^{-1} d c^{\#}, c e c\right) .
\end{aligned}
$$


Computations with $U$-operators (second construction): We now work with $A=J(B, \sigma, u, \mu)$, a second Tits construction Albert algebra. We have, for $x=$ $(a, b), y=(c, d) \in A$,

$$
\begin{gathered}
x^{\#}=\left(a^{\#}-b u \sigma(b), \bar{\mu} \sigma(b)^{\#} u^{-1}-a b\right), y^{\#}=\left(c^{\#}-d u \sigma(d), \bar{\mu} \sigma(d)^{\#} u^{-1}-c d\right), \\
T(x, y)=T_{B}(a c)+T_{B}(b u \sigma(d))+\overline{T_{B}(b u \sigma(d))}, \\
x \times y=\left(a \times c-b u \sigma(d)-d u \sigma(b), \bar{\mu}(\sigma(b) \times \sigma(d)) u^{-1}-a d-c b\right) .
\end{gathered}
$$

Hence we have $(a, 0)^{\#}=\left(a^{\#}, 0\right)$ and

$$
(a, 0)^{\#} \times(c, d)=\left(a^{\#}, 0\right) \times(c, d)=\left(a^{\#} \times c,-a^{\#} d\right) .
$$

Now,

$$
U_{x}(y)=T(x, y) x-x^{\#} \times y .
$$

For $x, y \in \mathcal{H}(B, \sigma)$ we note that $U_{x}(y)=x y x$. Therefore we have

$$
\begin{aligned}
U_{(a, 0)}(c, d) & =T((a, 0),(c, d))(a, 0)-(a, 0)^{\#} \times(c, d) \\
& =T_{B}(a c)(a, 0)-\left(a^{\#} \times c,-a^{\#} d\right) \\
& =\left(T_{B}(a c) a-a^{\#} \times c, a^{\#} d\right) \\
& =\left(U_{a}(c), a^{\#} d\right) \\
& =\left(a c a, a^{\#} d\right) .
\end{aligned}
$$

Now we come to the most crucial part of this paper. We will prove some key results, which will facilitate the proof of the Tits-Weiss conjecture. First we dispose of a necessary basic computation:

Theorem 5.5. Let $A=J(D, \mu)$ be a first Tits construction Albert algebra and $p \in S L(1, D)$ be a commutator in $D^{*}$. Then $\mathcal{J}_{p}$ is inner.

Proof. Recall that $\mathcal{J}_{p}: J(D, \mu) \longrightarrow J(D, \mu)$ is given by $\mathcal{J}_{p}(x, y, z)=\left(x, y p, p^{-1} z\right)$. Since $p$ is a commutator in $D^{*}$, we can write $p=j i j^{-1} i^{-1}$ for $i, j \in D^{*}$. Hence $j i j^{-1}=p i$, i.e. $j i=p i j$ and $j i^{-1} j^{-1}=i^{-1} p^{-1}$. Using the formulae that we derived on $U$ operators, we have, in $J(D, \mu)$,

$$
\begin{aligned}
U_{\left((i j)^{-1}, 0,0\right)} U_{(i, 0,0)} U_{(j, 0,0)} U_{(0,1,0)}(x, y, z) & =U_{\left((i j)^{-1}, 0,0\right)} U_{(i, 0,0)} U_{(j, 0,0)}(\mu y, z, \mu x) \\
& =U_{\left((i j)^{-1}, 0,0\right)} U_{(i, 0,0)}\left(j \mu y j, j^{\#} z, \mu x j^{\#}\right) \\
& =U_{\left((i j)^{-1}, 0,0\right)}\left(i j \mu y j i, i^{\#} j^{\#} z, \mu x j^{\#} i^{\#}\right) .
\end{aligned}
$$

Now,

$$
\begin{aligned}
U_{\left((i j)^{-1}, 0,0\right)} & \left(i j \mu y j i, i^{\#} j^{\#} z, \mu x j^{\#} i^{\#}\right) \\
& =\left((i j)^{-1} i j \mu y j i(i j)^{-1},\left(j^{-1} i^{-1}\right)^{\#} i^{\#} j^{\#} z, \mu x j^{\#} i^{\#}\left(j^{-1} i^{-1}\right)^{\#}\right) \\
& =\left(\mu y j i j^{-1} i^{-1}, i^{\#-1} j^{\#-1} i^{\#} j^{\#} z, \mu x\right) \\
& =\left(\mu y p,\left(j i j^{-1} i^{-1}\right)^{\#} z, \mu x\right) \\
& =\left(\mu y p, p^{-1} z, \mu x\right) .
\end{aligned}
$$

Finally we have,

$$
U_{(0,0,1)}\left(\mu y p, p^{-1} z, \mu x\right)=\left(\mu^{-1} \mu x, \mu^{-1} \mu y p, p^{-1} z\right)=\left(x, y p, p^{-1} z\right)=\mathcal{J}_{p}(x, y, z) .
$$

It follows that $\mathcal{J}_{p}$ is inner. 
Reduced Whitehead groups: Let $D$ be a central simple algebra over a field $k$. Let $S L(1, D)=\{x \in D \mid N r d(x)=1\}$ and $D^{*}=\{x \in D \mid N r d(x) \neq 0\}$. Then in $D^{*}$, the commutator subgroup $\left(D^{*}, D^{*}\right) \subset S L(1, D)$. The reduced Whitehead group of $D$ is defined by

$$
S K(1, D)=S L(1, D) /\left(D^{*}, D^{*}\right) .
$$

We have the following result of Wang ([33]) (see also [30], Proposition 2.7):

Theorem 5.6. Let $D$ be a central simple algebra over $k$ of square-free index. Then $S K(1, D)=0$. In particular, when the degree of $D$ is a prime, $S K(1, D)=0$.

Corollary 5.3. Let $D$ be a central division algebra of degree 3 over $k$ and let $p \in S L(1, D)$. Let $J(D, \mu)$ be a first Tits construction Albert algebra over $k$. Then $\mathcal{J}_{p}: J(D, \mu) \longrightarrow J(D, \mu)$ is inner.

Proof. Observe that for $p_{1}, \ldots, p_{r} \in S L(1, D), \mathcal{J}_{p_{1} \cdots p_{r}}=\mathcal{J}_{p_{r}} \cdots \mathcal{J}_{p_{1}}$, and that $\left(D^{*}, D^{*}\right)=S L(1, D)$. Hence the assertion follows from Theorem 5.5 .

Reduced unitary Whitehead groups: Let $(B, \tau)$ be a central simple algebra with a unitary involution $\tau$ over a field $k$. Let $\Sigma_{\tau}(B)$ be the subgroup of $B^{*}$ generated by $\operatorname{Sym}(B, \tau)^{*}$, where

$$
\operatorname{Sym}(B, \tau)=\left\{x \in B^{*} \mid \tau(x)=x\right\} .
$$

Let $\Sigma_{\tau}^{\prime}(B)$ be defined by

$$
\Sigma_{\tau}^{\prime}(B)=\left\{x \in B^{*} \mid N_{B}(x) \in k^{*}\right\} .
$$

Clearly $\Sigma_{\tau}^{\prime}(B)$ is a subgroup of $B^{*}$ and $\Sigma_{\tau}(B) \subset \Sigma_{\tau}^{\prime}(B)$. The reduced unitary Whitehead group of $B$ is defined by

$$
U S K_{1}(B)=\Sigma_{\tau}^{\prime}(B) / \Sigma_{\tau}(B) .
$$

We have the following analogue of Wang's result, due to Yanchevskii (see [10, Prop. 17.27):

Proposition 5.1. If $B$ is a central division algebra of prime degree with a unitary involution, then $U S K_{1}(B)=0$.

We now derive an analogue of Corollary 5.3 for second Tits constructions.

Corollary 5.4. Let $(B, \sigma)$ be a central division algebra of degree 3 over a quadratic extension $K / k$ with a unitary involution and let $p \in S U(B, \sigma)$. Let $J(B, \sigma, u, \mu)$ be a second Tits construction Albert algebra over $k$. Then $\phi_{p}: J(B, \sigma, u, \mu) \longrightarrow$ $J(B, \sigma, u, \mu)$ is inner.

Proof. Recall that $\phi_{p}: J(B, \sigma, u, \mu) \longrightarrow J(B, \sigma, u, \mu)$ is given by $\phi_{p}(a, b)=(\operatorname{pa\sigma }(p)$, $p b)$. Since $p \in S U(B, \sigma)$, we have $p \sigma(p)=1$ and $N_{B}(p)=p p^{\#}=\sigma(p)^{\#} \sigma(p)=1$. By Proposition 5.1 we can write $p=s_{1} s_{2} \cdots s_{n}$ for some $s_{1}, \ldots, s_{n} \in \operatorname{Sym}(B, \sigma)$. Hence $\sigma(p)=s_{n} s_{n-1} \cdots s_{1}$ and we have

$$
\begin{aligned}
\phi_{p}(a, b)=(p a \sigma(p), p b) & =\left(s_{1} \cdots s_{n} a \sigma\left(s_{1} \cdots s_{n}\right), \sigma(p)^{-1} b\right) \\
& =\left(s_{1} \cdots s_{n} a s_{n} \cdots s_{1}, \sigma(p)^{\#} b\right) \\
& =\left(s_{1} \cdots s_{n} a s_{n} \cdots s_{1},\left(s_{n} \cdots s_{1}\right)^{\#} b\right) \\
& =\left(s_{1} s_{2} \cdots s_{n} a s_{n} s_{n-1} \cdots s_{1}, s_{1}^{\#} s_{2}^{\#} \cdots s_{n}^{\#} b\right) \\
& =U_{\left(s_{1}, 0\right)} \cdots U_{\left(s_{n}, 0\right)}(a, b),
\end{aligned}
$$


where the last line follows from the computations with $U$-operators in the case of second Tits constructions done above. Hence $\phi_{p}$ is inner.

Theorem 5.7. Let $A$ be an Albert algebra arising from the first Tits construction. Let $\phi \in \operatorname{Aut}(A)$ be an automorphism of $A$ that fixes pointwise a subalgebra of the form $D_{+}$for a degree 3 central simple algebra $D$ over $k$. Then $\phi$ is inner.

Proof. The inclusion $D_{+} \hookrightarrow A$ induces an isomorphism $\theta: A \longrightarrow J(D, \mu)$ for some $\mu \in k^{*}$ such that $\theta\left(D_{+}\right)=D_{0}$. Let $\psi=\theta \phi \theta^{-1}: J(D, \mu) \longrightarrow J(D, \mu)$. Then $\psi$ fixes $D_{0}$ pointwise. Hence $\psi$ is given by $\psi(x, y, z)=\left(x, y p, p^{-1} z\right)$ for some $p \in S L(1, D)$. Now, by the above theorem of Wang, $S K(1, D)=0$ if degree of $D$ is a prime. Hence $\psi=\mathcal{J}_{p}$ for some $p \in S L(1, D)$ and thus $\psi$ is inner, by Corollary 5.3. Hence there are elements $a_{1}, \ldots, a_{r} \in J(D, \mu)$ such that $\psi=U_{a_{1}} \cdots U_{a_{r}}$. Therefore we have $\phi=\theta^{-1} U_{a_{1}} \cdots U_{a_{r}} \theta$. Now, for $a \in J(D, \mu), U_{a}=2 R_{a}^{2}-R_{a^{2}}$. Hence, for any $x \in A$,

$$
\begin{aligned}
\theta^{-1} U_{a} \theta(x) & =\theta^{-1}\left(2 R_{a}^{2}-R_{a^{2}}\right)(\theta(x))=2 \theta^{-1}(a(a \theta(x)))-\theta^{-1}\left(a^{2} \theta(x)\right) \\
& =\left[2 R_{\theta^{-1}(a)}^{2}-R_{\theta^{-1}(a)^{2}}\right](x)=U_{\theta^{-1}(a)}(x) .
\end{aligned}
$$

Hence we have, $\phi=U_{\theta^{-1}\left(a_{1}\right)} \cdots U_{\theta^{-1}\left(a_{r}\right)}$.

Corollary 5.5. Let $A$ be a pure first construction Albert division algebra over $k$. Then every automorphism of $A$ is inner.

Proof. This follows from Corollary 5.2 . Theorem 5.4, and Theorem 5.7

We will now prove that cubes of norm 1 elements of a degree 3 central division algebra are products of two commutators. To prove this we need a version of the Wedderburn Factorization Theorem for degree 3 division algebras (see [8], Lemma 2.9.8). This gives an easy proof of the fact that for $A=J(D, \mu)$ and $p \in S L(1, D)$ the automorphism $\mathcal{J}_{p}^{3}$ is inner. This of course is a consequence of Corollary 5.3 proved above, but the proof we present below avoids the use of triviality of $S K(1, D)$; moreover, it yields a more precise statement; cf. Proposition 5.3 below. A little terminology first:

Definition. We call an element $a$ of a division algebra $D$ over $k$ cyclic if $k(a)$ is a cyclic subfield of $D$.

Proposition 5.2 (Wedderburn Factorization Theorem). Let $D$ be a central division algebra of degree 3 over $k$ and let $a \in D^{*}$ be a noncyclic element. Then the minimum polynomial $f(X)=X^{3}-\alpha_{1} X^{2}+\alpha_{2} X-\alpha_{3}$ of a over $k$ has a factorization

$$
f(X)=\left(X-a_{2}\right)\left(X-a_{1}\right)\left(X-a_{0}\right)
$$

in $D[X]$ with $a_{0}=a$,

$$
c=\left[a_{0} a_{1}\right]=\left[a_{1} a_{2}\right]=\left[a_{2} a_{0}\right] \neq 0, c a_{i} c^{-1}=a_{i+1},
$$

where the indices are reduced modulo 3 . Further $c^{3}=\gamma \in k^{*}$. Here $[x y]=x y-$ $y x, x, y \in D$.

We have

Proposition 5.3. Let $D$ be a degree 3 central division algebra over $k$ and $p \in$ $S L(1, D)$. Then $p^{3}$ is a product of two commutators in $D^{*}$. 
Proof. First let us assume $p \in S L(1, D)$ is cyclic. Then $L=k(p)$ is a cyclic cubic subfield of $D$. Hence $L$ is a maximal subfield of $D$ and $N_{D}(p)=N_{L / k}(p)=1=$ $N_{L / k}\left(p^{3}\right)$. Let $\operatorname{Gal}(L / k)=\langle\sigma\rangle$. Then, by Hilbert Theorem 90, there exists $q \in L$ such that $p^{3}=q^{-1} \sigma(q)$. By the Skolem-Noether theorem, we can extend $\sigma$ to an automorphism of $D$. Hence there exists $x \in D^{*}$ such that $\sigma(q)=x q x^{-1}$. Therefore we have

$$
p^{3}=q^{-1} \sigma(q)=q^{-1} x q x^{-1} .
$$

Now assume $p \in S L(1, D)$ is noncyclic. Let $p=a=p_{0}$ in the proposition above. Then there are $p_{1}, p_{2}, c \in D^{*}$ such that the minimal polynomial $f(X)=X^{3}-$ $\alpha_{1} X^{2}+\alpha_{2} X-\alpha_{3}$ of $p_{0}$ over $k$ factorizes in $D[X]$ as

$$
f(X)=\left(X-p_{2}\right)\left(X-p_{1}\right)\left(X-p_{0}\right)
$$

and we have the relations

$$
c p_{0} c^{-1}=p_{1}, c p_{1} c^{-1}=p_{2}, c p_{2} c^{-1}=p_{0} .
$$

It follows that $p_{i} \in S L(1, D), i=0,1,2$. From these relations, we get

$$
c p_{0} c^{-1} p_{0}^{-1}=p_{1} p_{0}^{-1}, c p_{1} c^{-1} p_{1}^{-1}=p_{2} p_{1}^{-1}, c p_{2} c^{-1} p_{2}^{-1}=p_{0} p_{2}^{-1} .
$$

Therefore the elements $p_{1} p_{o}^{-1}, p_{2} p_{1}^{-1}, p_{0} p_{2}^{-1}$ are commutators. Expanding $f(X)$ and using the fact that $N_{D}(p)=1$, we get $p_{2} p_{1} p_{0}=1$. Hence we have the relations

$$
p_{1} p_{0}=p_{2}^{-1}, p_{2} p_{1}=p_{0}^{-1}, p_{0} p_{2}=p_{1}^{-1} \text {. }
$$

Now $p_{0} p_{1}^{-1}=\left(p_{1} p_{0}^{-1}\right)^{-1}$ is a commutator; hence substituting $p_{1}^{-1}=p_{0} p_{2}$ we get that $p_{0}\left(p_{0} p_{2}\right)=p_{0}^{2} p_{2}$ is also a commutator. By the conjugacy relations above, we have $c p_{2} c^{-1}=p_{0}$; hence $p_{2}^{-1} c p_{2} c^{-1}=p_{2}^{-1} p_{0}$, and hence $p_{2}^{-1} p_{0}$ is a commutator. It follows that $\left(p_{0}^{2} p_{2}\right)\left(p_{2}^{-1} p_{0}\right)=p_{0}^{3}$ is a product of two commutators in $D^{*}$. This proves the assertion.

Corollary 5.6. Let $p \in S L(1, D)$. Then $\mathcal{J}_{p^{3}}=\left(\mathcal{J}_{p}\right)^{3}$ is inner.

Proof. This is immediate now from Theorem 5.5.

We have the following for arbitrary Tits first construction Albert division algebras.

Theorem 5.8. Let $A=J(D, \mu)$ be a first Tits construction Albert division algebra. Let $a \in D^{*}$. Then $\mathcal{I}_{a}: J(D, \mu) \longrightarrow J(D, \mu)$ given by $\mathcal{I}_{a}(x, y, z)=$ $\left(a x a^{-1}, a y a^{-1} a z a^{-1}\right)$ is inner.

Proof. We calculate with $a$ as follows:

$$
\begin{aligned}
& U_{(0, a, 0)} U_{(0,0, a)} U_{(a, 0,0)} U_{(0,1,0)}(x, y, z)=U_{(0, a, 0)} U_{(0,0, a)} U_{(a, 0,0)}(\mu y, z, \mu x) \\
& \quad=U_{(0, a, 0)} U_{(0,0, a)}\left(\mu a y a, n(a) a^{-1} z, \mu n(a) x a^{-1}\right) \\
& \quad=U_{(0, a, 0)}\left(n(a)^{2} a^{-1} x a^{-1}, n(a) a y, n(a) z a\right) \\
& \quad=\left(\mu n(a)^{2} a y a^{-1}, n(a) a z a^{2}, \mu n(a)^{3} a^{-2} x a^{-1}\right) .
\end{aligned}
$$


We now note that $n(a) a^{-3} \in S L(1, D)$. Hence $\mathcal{J}_{n(a) a^{-3}}$ is inner by Corollary 5.3. We apply the transformation $U_{n(a)^{-1}} U_{(0,0,1)} \mathcal{J}_{n(a) a^{-3}}$ to the above and get

$$
\begin{aligned}
U_{n(a)^{-1}} U_{(0,0,1)} \mathcal{J}_{n(a) a^{-3}}\left(\mu n(a)^{2} a y a^{-1}, n(a) a z a^{2}, \mu n(a)^{3} a^{-2} x a^{-1}\right) \\
=n(a)^{-2} U_{(0,0,1)}\left(\mu n(a)^{2} a y a^{-1}, n(a)^{2} a z a^{-1}, \mu n(a)^{2} a x a^{-1}\right) \\
=n(a)^{-2}\left(n(a)^{2} a x a^{-1}, n(a)^{2} a y a^{-1}, n(a)^{2} a z a^{-1}\right) \\
=\left(a x a^{-1}, a y a^{-1}, a z a^{-1}\right)=\mathcal{I}_{a}(x, y, z) .
\end{aligned}
$$

This proves the assertion.

Corollary 5.7. Let $A$ be a first Tits construction Albert division algebra over $k$ and let $\psi \in A u t(A)$ be such that $\psi\left(D_{+}\right)=D_{+}$for a subalgebra $D_{+} \subset A$. Then $\psi$ is an inner automorphism of $A$.

Proof. As in arguments seen before, we may assume $A=J(D, \mu)$ for a suitable scalar $\mu \in k^{*}$ and $D_{+}=D_{0}$. Hence we have $\psi \in \operatorname{Aut}(A)$ such that $\psi\left(D_{0}\right)=D_{0}$. Then it follows that $\psi=\psi_{a, b}$ for some $a, b \in D^{*}$ with $N_{D}(a)=N_{D}(b)$. We have

$$
\psi(x, y, z)=\left(a x a^{-1}, a y b^{-1}, b z a^{-1}\right)=\mathcal{J}_{a b^{-1}} \mathcal{I}_{a}(x, y, z) .
$$

Hence $\psi=\mathcal{J}_{a b^{-1}} \mathcal{I}_{a}$ and we have seen that $\mathcal{I}_{a} \in \operatorname{Instr}(A)$. Since $a b^{-1} \in S L(1, D)$, $\mathcal{J}_{a b^{-1}} \in \operatorname{Instr}(A)$. Hence the assertion follows.

Notation. We need some notation at this stage. Let $A=J(D, \mu)$ be a first Tits construction Albert division algebra over $k$ and $S \subset A$ a subalgebra. We will denote by $C$ the subgroup of $\operatorname{Str}(A)$ consisting of scalar multiplications on $A$. We will abuse notation and will denote by $N_{S}$ the subgroup of $C$ consisting of scalar multiplications on $A$ by norms from $D^{*}$.

The following lemma and its corollary clarify the action of $\operatorname{Str}(A)$ on $A^{*}$.

Lemma 5.1. Let $A=J(D, \mu)$ be a first Tits construction Albert division algebra and $a \in D^{*}$. Then

$$
\chi=R_{N_{D}(a)^{-1}} U_{(0,0,1)} U_{(0, a \#, 0)} \in N_{D} \cdot \operatorname{Instr}(A)
$$

stabilizes $D_{0} \subset A$ and maps $(1,0,0)$ to $(a, 0,0)$.

Proof. We have for $\chi=R_{N_{D}(a)^{-1}} U_{(0,0,1)} U_{\left(0, a a^{\#}, 0\right)}$,

$$
\begin{aligned}
\chi(1,0,0) & =R_{N_{D}(a)^{-1}} U_{(0,0,1)}\left(0,0, \mu N_{D}(a) a\right) \\
& =R_{N_{D}(a)^{-1}}\left(N_{D}(a) a, 0,0\right) \\
& =(a, 0,0) .
\end{aligned}
$$

Moreover, for $x \in D$,

$$
\begin{aligned}
\chi(x, 0,0) & =N_{D}(a)^{-1} U_{(0,0,1)} U_{(0, a \#, 0)}(x, 0,0) \\
& =N_{D}(a)^{-1} U_{(0,0,1)}\left(0,0, \mu a^{\# \#} x\right) \\
& =N_{D}(a)^{-1}\left(\mu^{-1} \mu N_{D}(a) a x, 0,0\right) \\
& =(a x, 0,0) .
\end{aligned}
$$

Hence $\chi\left(D_{0}\right)=D_{0}$ as asserted. 
Corollary 5.8. Let $A$ be a pure Tits first construction Albert algebra and $a$ an invertible element of $A$. Then there exists $\chi \in C$.Instr $(A)$ such that $\chi(1)=a$.

Proof. Let us imbed $a$ in a subalgebra of $A$ of the form $D_{+}$for a degree 3 central division algebra over $k$. Let $\theta: A \longrightarrow J(D, \mu)$ be an isomorphism extending the inclusion $D_{+} \hookrightarrow A$ for some $\mu \in k^{*}$ and $\theta\left(D_{+}\right)=D_{0}$. Let $\chi^{\prime} \in C \cdot \operatorname{Instr}(J(D, \mu))$ be as in the lemma above. Then $\chi^{\prime}(1,0,0)=(a, 0,0)$. Let $\chi=\theta^{-1} \chi^{\prime} \theta: A \longrightarrow A$. Then

$$
\chi(1)=\theta^{-1} \chi^{\prime} \theta(1)=\theta^{-1} \chi^{\prime}(1,0,0)=\theta^{-1}(a, 0,0)=a .
$$

Further,

$$
\chi=\theta^{-1} \chi^{\prime} \theta=\theta^{-1} R_{N_{D}(a)^{-1}} U_{(0,0,1)} U_{(0, a \#, 0)} \theta=R_{N_{D}(a)^{-1}} U_{\theta^{-1}(0,0,1)} U_{\theta^{-1}(0, a \#, 0)} .
$$

Hence $\chi \in C$.Instr $(A)$.

The theorem below tells us about the norm similarities of an Albert algebra that stabilize a 9-dimensional subalgebra.

Theorem 5.9. Let $A$ be a first Tits construction Albert algebra. Let $\psi \in \operatorname{Str}(A)$ be such that $\psi\left(D_{+}\right)=D_{+}$for a subalgebra $D_{+} \subset A$. Then $\psi \in N_{D} \operatorname{Instr}(A)$.

Proof. By a simple argument, we may assume that $A=J(D, \mu)$ and identify $D_{+}$ with $D_{0} \subset J(D, \mu)$. Let $\psi \in \operatorname{Str}(A)$ be such that $\psi\left(D_{0}\right)=D_{0}$. In particular $\psi(1,0,0)=(c, 0,0)$ for some $c \in D^{*}$. Let $\chi \in N_{D}$.Instr $(A)$ be as in the lemma above. Then $\chi\left(D_{0}\right)=D_{0}$ and $\chi(1,0,0)=(c, 0,0)$. Moreover, we have

$$
\chi^{-1} \psi(1,0,0)=\chi^{-1}(c, 0,0)=(1,0,0) .
$$

Therefore $\phi=\chi^{-1} \psi \in A u t(A)$ and $\phi\left(D_{0}\right)=D_{0}$. Hence $\phi=\psi_{a, b}$ for suitable $a, b \in D^{*}$. We have proved already that $\psi_{a, b} \in \operatorname{Instr}(A)$ (see Corollary [5.7). Hence $\psi=\chi \psi_{a, b} \in N_{D} \operatorname{Instr}(A)$.

In the spirit of the above theorem, combining Theorem 5.3 with Lemma 5.1 gives

Theorem 5.10. Let $A$ be an Albert division algebra over $k$ arising from the first Tits construction. Let $E \subset A$ be a cubic cyclic extension of $k$. Let $\psi \in \operatorname{Str}(A)$ be such that $\psi(E)=E$. Then $\psi \in C$.Instr $(A) . H$, where $H$ denotes the subgroup of Aut $(A)$ generated by automorphisms, each stabilizing a 9-dimensional subalgebra of A.

Proof. Since $E / k$ is a cyclic cubic extension, there is a 9-dimensional subalgebra $D_{+} \subset A$ such that $E \subset D_{+}$. We may assume (see proof of Theorem [5.7), that $A=J(D, \mu)$ for some $\mu \in k^{*}$. Hence we may identify $D_{+}$with $D_{0} \subset A$ and $E \subset D_{0}$. Let $\psi(1)=\psi(1,0,0)=(c, 0,0), c \in E$. Let $\chi=R_{N_{E}(c)^{-1}} U_{(0,0,1)} U_{\left(0, c^{\#}, 0\right)} \in$ $N_{D} \operatorname{Instr}(A)$. Then we have, by a computation exactly as in the proof of Lemma 5.1 .

$$
\chi(1,0,0)=(c, 0,0)=\psi(1,0,0) .
$$

Note also $\chi(E)=E$; in fact, for any $x \in E, \chi(x, 0,0)=(c x, 0,0)$. This follows by a computation along the lines of the proof of Lemma 5.1. We have therefore 
$\chi^{-1} \psi(1,0,0)=(1,0,0)$ and hence $\chi^{-1} \psi \in A u t(A)$ with $\chi^{-1} \psi(E)=E$. Therefore, by Corollary 5.1, it follows that $\chi^{-1} \psi \in H$. Hence $\psi \in C$.Instr $(A)$.H.

In light of these two theorems, one can raise the following question:

Question. Let $A$ be an Albert division algebra over $k$, arising from the first construction. Let $D_{+} \subset A$ and $X=\operatorname{Str}(A)\left(D_{+}\right)$be the orbit of $D_{+}$under the structure group. Does the subgroup $C$.Instr $(A)$ act transitively on $X$ ? What if we consider the orbit $Y=\operatorname{Str}(A)(E)$ of a cubic cyclic subfield of $A$ ?

Remark. An affirmative answer to the first would prove the Tits-Weiss conjecture for first constructions, while an affirmative answer to the second would prove that for first constructions, any norm similarity is a product of an element of $C$.Instr $(A)$ and an element of $H$.

For pure first constructions, we have a stronger result:

Theorem 5.11 (Tits-Weiss Conjecture). Let $A$ be a pure first construction Albert division algebra over a field $k$. Then

$$
\frac{\operatorname{Str}(A)}{\operatorname{C.Instr}(A)}=\{1\} .
$$

Proof. Let $\phi \in \operatorname{Str}(A)$, where $A$ is a pure first Tits construction Albert division algebra over $k$. Let $\phi(1)=a$. Then, by Corollary [5.8, there exists $\chi \in C$.Instr $(A)$ such that $\chi(a)=1$. Hence $\chi \phi(1)=1$. This shows that $\chi \phi$ is an automorphism of $A$. Hence we have reduced the problem to proving that every automorphism of a pure first Tits construction Albert division algebra is inner. This has already been proved! (See Corollary [5.5])

The case of reduced Albert algebras: One can discuss the Tits-Weiss conjecture in the setting of reduced Albert algebras as well. As we shall see, in this case, it follows rather easily from known results of Jacobson that the assertion of the conjecture is indeed true. This doesn't seem to be explicit in the literature. We therefore record it below. In the following discussion, we will denote by $\operatorname{Isom}(A)$ the subgroup of $\operatorname{Str}(A)$ consisting of all isometries of the norm $N$ of $A$.

Theorem 5.12. Let $A$ be a reduced Albert algebra over a field $k$. Then $\operatorname{Str}(A)=$ C.Instr $(A)$.

Proof. Let $\psi \in \operatorname{Str}(A)$ and $\psi(1)=a$. Let $\alpha=N(a)$. Then $\alpha \neq 0$ and $U_{a}$ is invertible. Consider $\chi=R_{\alpha^{-1}} U_{a} \psi \in C$.Instr $(A)$. Then we have

$$
\chi(1)=\alpha^{-1} U_{a} \psi(1)=\alpha^{-1} U_{a}(a)=\alpha^{-1} a^{3} .
$$

Hence,

$$
N(\chi(1))=\alpha^{-3} N\left(a^{3}\right)=\alpha^{3} N(a)^{3}=\alpha^{-3} \alpha^{3}=1 .
$$

It follows that $\chi \in I \operatorname{som}(A)$. It now suffices to prove that isometries of $N$ are inner. When $A$ is split, this follows from [9, Theorem 9], which proves that the norm-preserving group $\operatorname{Isom}(A)$ coincides with the subgroup of $\operatorname{Str}(A)$ consisting of all mappings $\eta=U_{a_{1}} \cdots U_{a_{r}}$ with $\prod N\left(a_{i}\right)=1$. If the coordinate algebra of $A$ is a division algebra, this follows from [6. Theorem 13], which proves that $\operatorname{Isom}(A)$ coincides with the subgroup of $\operatorname{Str}(A)$ generated by elements $\zeta=\prod U_{a_{i}}$, where the $a_{i}$ for a given $\zeta$ are all contained in some nonsplit 9-dimensional reduced simple subalgebra of degree 3 and $\prod N\left(a_{i}\right)=1$. 
$R$-equivalence in $k$-forms of $F_{4}$ : For the notion of $R$-equivalence, we refer to the book ([32, Chapter 6$)$ or the book ([11], Chapter II, 14). Let $X$ be an irreducible variety over a field $k$, with $X(k)$ nonempty. Manin in [11] introduced the notion of $R$-equivalence on $X$; call points $x, y \in X(k) R$-equivalent if there exist points $x=x_{0}, x_{1}, \ldots, x_{n}=y \in X(k)$ and rational maps $f_{i}: \mathbb{A}^{1} \rightarrow X, 1 \leq i \leq n$, all defined over $k$ and regular at 0 and 1 , such that $f_{i}(0)=x_{i-1}, f_{i}(1)=x_{i}$. In case $X=G$, a connected algebraic group, the set of elements of $G(k)$ which are $R$-equivalent to $1 \in G(k)$ is a normal subgroup $R G(k)$ of $G(k)$. The quotient $G(k) / R G(k)$ is denoted by $G(k) / R$. We now prove

Theorem 5.13. Let $A$ be a pure first construction Albert division algebra over a field $k$ and let $G=\operatorname{Aut}(A)$. Then we have

$$
G(k) / R=\{1\} .
$$

Proof. We have shown in Theorem 5.2 and Theorem 5.4 that every automorphism $\phi$ of a pure first construction Albert division algebra is a product of automorphism fixing 9-dimensional subalgebras of $A$. Now, every automorphism of $A$ that fixes a 9 -dimensional subalgebra, belongs to a $k$-subgroup of type $A_{2}$ (see Proposition 4.2). By [2], groups of rank 2 are rational, hence $R$-trivial. Therefore each factor of $\phi$ can be connected to the identity automorphism by a rational map over $k$ as above. From this the result follows.

\section{ACKNOWLEDGEMENTS}

The author would like to express his deepest gratitude to H. P. Petersson for meticulously reading the preliminary version of this paper and pointing out several errors. The author also thanks Richard Weiss, Tom De Medts, B. Sury and Dipendra Prasad for generously sharing their knowledge with him on the subject. Katrin Tent and Linus Kramer supported the author's visit to the University of Wuerzburg back in 2004, where this work was conceived; their help is gratefully acknowledged. The author thanks the Abdus Salam I.C.T.P., Trieste, for its hospitality on various occasions. The author thanks Amit Kulshrestha, Anupam Singh and Shripad Garge for their interest in the work and would also like to thank G. Prasad for supplying the reference [30] for the result of Wang. The author thanks the referee for a very encouraging report on this work.

\section{REFERENCES}

[1] A. Borel, Linear Algebraic Groups, Graduate Texts in Math. 126, Springer-Verlag, New York, 1991. MR1102012(92d:20001)

[2] Vladimir I. Chernousov and Vladimir P. Platonov, The rationality problem for semisimple group varieties, J. Reine Angew. Math. 504 (1998), 1-28. MR1656830 (99i:14056)

[3] J. R. Faulkner and J. C. Ferrar, Exceptional Lie Algebras and Related Algebraic and Geometric Structures, Bull. London Math. Soc. 9 (1977), 1-35. MR0444729 (56:3079)

[4] Philippe Gille, Le Problème de Kneser-Tits, Séminaire Bourbaki 982 (2007), Astérisque 326 (2009), 39-81. MR2605318(2011h:20099)

[5] J. E. Humphreys, Conjugacy Classes in Semisimple Algebraic Groups, Mathematical Surveys and Monographs, Volume 43, AMS, Providence, RI,1995. MR1343976 (97i:20057)

[6] N. Jacobson, Structure and representations of Jordan algebras, Colloquium Publications, Vol. XXXIX. AMS, Providence, R. I., 1968. MR.0251099 (40:4330)

[7] N. Jacobson, Composition algebras and their automorphisms, Rend. Circ. Mat. Palermo (2) 7 (1958) 55-80. MR0101253 (21:66) 
[8] N. Jacobson, Finite-dimensional division algebras over fields, Springer-Verlag, Berlin, 1996. MR $1439248(98 \mathrm{a}: 16024)$

[9] N. Jacobson, Some groups of transformations defined by Jordan Algebras. III. Groups of type $E_{6}$, J. reine angew. Math. 207 (1961), 61-85. MR0159850(28:3066)

[10] M. A. Knus, A. Merkurjev, M. Rost, and J. P. Tignol, The Book of Involutions, AMS. Colloquium Publications, Vol. 44, 1998. MR1632779 (2000a:16031)

[11] Yu. I. Manin, Cubic forms, North-Holland, Amsterdam, 1974. MR833513 (87d:11037)

[12] H. P. Petersson, Structure theorems for Jordan algebras of degree three over fields of arbitrary characteristic, Comm. Algebra 32 (2004), no. 3, 1019-1049. MR2063796 (2005h:17061)

[13] H. P. Petersson and M. Racine, Pure and generic first Tits constructions of exceptional Jordan division algebras, Algebras, Groups and Geometries 3 (1986), 386-398. MR900490 (88h:17033)

[14] H. P. Petersson and M. Racine, Springer forms and the first Tits construction of exceptional Jordan division algebras, Manuscripta Math. 45 (1984), no. 3, 249-272. MR734841 (85i:17029)

[15] H. P. Petersson and M. Racine, An elementary approach to the Serre-Rost invariant of Albert algebras, Indag. Mathem., N.S. 7 (1996), no. 3, 343-365. MR1621373 (99j:17045)

[16] H. P. Petersson and M. Racine, Radicals of Jordan algebras of degree 3, Radical theory (Eger, 1982), Colloq. Math. Soc. Janos Bolyai, Vol. 38, North-Holland, Amsterdam, 1985, 349-377. MR899120 (88g:17028)

[17] H. P. Petersson and M. Racine, Albert algebras, Jordan algebras (Oberwolfach, 1992)(W. Kaup and McCrimmon, eds.), de Gruyter, Berlin, 1994, no. 3, 197-207. MR.1293320 (95k:17043)

[18] H. P. Petersson and M. Racine, Classification of algebras arising from the Tits process, J. Algebra 98 (1986), no. 1, 244-279. MR825145 (87h:17038b)

[19] V. P. Platonov and A. Rapinchuk, Algebraic groups and number theory, Pure and Applied Mathematics, Vol. 139, Academic Press Inc., Boston, MA, 1994. MR.1278263 (95b:11039)

[20] R. Parimala, V. Suresh and Maneesh L. Thakur, Jordan Algebras and $F_{4}$ Bundles over the Affine Plane, J. Algebra 198 (1997), 582-607. MR.1489913 (98k:17035)

[21] R. W. Richardson, Conjugacy classes in Lie algebras and algebraic groups, Annals of Math. Second Series, Vol. 86, No. 1 (1967), 1-15. MR0217079 (36:173)

[22] J. P. Serre, Galois Cohomology, Springer-Verlag, 1994. MR1324577 (96b:12010)

[23] T. A. Springer and F. D. Veldkamp, Octonions, Jordan Algebras and Exceptional Groups, Springer Monographs in Mathematics, Springer-Verlag, Berlin, 2000. MR1763974 (2001f:17006)

[24] T. A. Springer, Linear Algebraic Groups, Second Edition, Progress in Mathematics, Birkhäuser, Boston, 1998. MR1642713(99h:20075)

[25] Tom De Medts and Hendrik Van Maldeghem, Moufang Generalized Polygons, Topics in Diagram Geometry, Quaderni di Matematica, Volume 12 (edited by Antonio Pasini), 59-126, Dept. Math., Seconda Univ. Napoli, Caserta, 2003. MR2066523 (2005d:51007)

[26] M. Thakur, Isotopy and invariants of Albert algebras, Comment. Math. Helv. 74 (1999), 297-305. MR1691951 (2000f:17046)

[27] J. Tits, Strongly inner anisotropic forms of simple algebraic groups, Journal of Alg. 131 (1990), 648-677. MR1058572 (91g:20069)

[28] J. Tits, Classification of buildings of spherical type and Moufang polygons: A Survey, Colloquio Internazionale sulle Teorie Combinatorie (Roma, 1973), Tomo I, pp. 229-246. Atti dei Convegni Lincei, No. 17, Accad. Naz. Lincei, Rome, 1976. MR0444793 (56:3140)

[29] J. Tits, Classification of algebraic semisimple groups, Algebraic Groups and Discontinuous Subgroups (Proc. Sympos. Pure Math., Boulder, Colo., 1965) (A. Borel and G. D. Mostow, eds.), Vol. 9, AMS., Providence, R.I., 1966, 33-62. MR0224710 (37:309)

[30] J. Tits, Groupes de Whitehead de groupes algébriques simples sur un corps, Seminaire Bourbaki, 1976-77, $n^{\circ} 505$, Lecture Notes in Mathematics 677, Springer-Verlag, New York, Heidelberg, Berlin, 1978. MR0521771 (80d:12008)

[31] J. Tits and R. M. Weiss, Moufang Polygons, Springer Monographs in Mathematics, SpringerVerlag, 2002. MR 1938841(2003m:51008)

[32] V. E. Voskresenskii, Algebraic Groups and Their Birational Invariants, Translation of Math. Monographs, Vol. 179, AMS, Providence, RI, 1998. MR1634406 (99g:20090) 
[33] S. Wang, On the commutator subgroup of a simple algebra, Amer. J. Math. 72 (1950), 323334. MR0034380 (11:577d)

[34] R. M. Weiss, The Structure of Spherical Buildings, Princeton University Press, 2003, Princeton. MR2034361 (2005b:51027)

[35] R. M. Weiss, Quadrangular Algebras, Princeton University Press, Mathematical Notes 46, Princeton University Press, 2006, Princeton. MR2177056 (2007a:17010)

[36] M. J. Wonenburger, Automorphisms of Cayley algebras, Journal of Algebra 12 (1969), 327338. MR0238917(39:277)

Indian Statistical Institute, 7-S.J.S. Sansanwal Marg, New Delhi 110016, India

E-mail address: maneesh.thakur@gmail.com 